\title{
Dynamics Analysis of a Stochastic Hybrid Logistic Model with Delay and Two-Pulse Perturbations
}

\author{
Haokun Qi (iD) and Hua Guo (iD) \\ College of Mathematics and Systems Science, Shandong University of Science and Technology, Qingdao 266590, China \\ Correspondence should be addressed to Hua Guo; skd993121@sdust.edu.cn
}

Received 11 May 2020; Accepted 1 July 2020; Published 17 August 2020

Guest Editor: Songbai Guo

Copyright ( $\odot 2020$ Haokun Qi and Hua Guo. This is an open access article distributed under the Creative Commons Attribution License, which permits unrestricted use, distribution, and reproduction in any medium, provided the original work is properly cited.

In this paper, we propose and discuss a stochastic logistic model with delay, Markovian switching, Lévy jump, and two-pulse perturbations. First, sufficient criteria for extinction, nonpersistence in the mean, weak persistence, persistence in the mean, and stochastic permanence of the solution are gained. Then, we investigate the lower (upper) growth rate of the solutions. At last, we make use of Matlab to illustrate the main results and give an explanation of biological implications: the large stochastic disturbances are disadvantageous for the persistence of the population; excessive impulsive harvesting or toxin input can lead to extinction of the population.

\section{Introduction}

It is universally known that the logistic model is one of the most significant and classical models in mathematical biology. Many scholars have studied it and achieved fruitful results (see [1-8]). The classical logistic equation is expressed by

$$
\mathrm{d} X(t)=X(t)\left[r-a_{1} X(t)\right] \mathrm{d} t
$$

where $X$ denotes the population size and $r$ and $a_{1}$ stand for the intrinsic growth rate and the intraspecific competition rate, respectively. With the improvement of the understanding of biological mathematical models, some factors have been considered, such as random interference, time delay, and so on. Compared with the classical original model, stochastic models (see [9-16]) can better reflect the actual situation. Based on model (1), we obtain the following stochastic model:

$$
\begin{aligned}
\mathrm{d} X(t)= & X(t)\left(r-a_{1} X(t)+a_{2} X(t-\tau)+a_{3} \int_{-\infty}^{0} X(t+\theta) \mathrm{d} \varsigma(\theta)\right) \mathrm{d} t \\
& +\sigma X(t) \mathrm{d} B(t)+X\left(t^{-}\right) \int_{\mathbb{V}} \gamma(v) \tilde{N}(\mathrm{~d} t, \mathrm{~d} v),
\end{aligned}
$$

where $r$ and $a_{i}(i=1,2,3)$ are non-negative constants. $\tau \geq 0$ is the time delay. $\varsigma(\theta)$ stands for a probability measure on $(-\infty, 0] . B(t)$ is the independent standard Brownian motion defined on a complete probability space $\left(\Omega, \mathscr{F},\left\{\mathscr{F}_{t}\right\}_{t \geq 0}, \mathbb{P}\right)$ and $\sigma \geq 0$ is the intensity of the white noise. $X\left(t^{-}\right)$is the left limit of $X(t) . N(\mathrm{~d} t, \mathrm{~d} v)$ is a Poisson random measure with characteristic measure $\lambda$ on a measurable bounded below

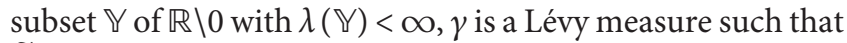
$\tilde{N}(\mathrm{~d} t, \mathrm{~d} v)=N(\mathrm{~d} t, \mathrm{~d} v)-\lambda(\mathrm{d} v) \mathrm{d} t$.

In addition to the white noise and Lévy noise mentioned above, there are other noises in nature, such as telegraph noise, which can be expressed by continuous-time Markov 
chain that mainly describes the random switching between two or more environment states [17] and which is different due to rainfall, nutrition, and other factors $[18,19]$. Thus, a series of articles about Markovian switching have been investigated (see [20-28]). We focus on the stochastic logistic model with Markovian switching:

$$
\begin{aligned}
\mathrm{d} X(t)= & X(t)\left[r(\xi(t))-a_{1}(\xi(t)) X(t)+a_{2}(\xi(t)) x(t-\tau)+a_{3}(\xi(t)) \int_{-\infty}^{0} X(t+\theta) \mathrm{d} \varsigma(\theta)\right] \mathrm{d} t \\
& +\sigma(\xi(t)) X(t) \mathrm{d} B(t)+X\left(t^{-}\right) \int_{\mathbb{V}} \gamma((\xi(t)), v) \tilde{N}(\mathrm{~d} t, \mathrm{~d} v),
\end{aligned}
$$

where $\xi(t)$ is a continuous-time Markov chain with values in finite state space $\mathbb{M}=\{1,2, \ldots, N\}$. We assume that $B(t)$ and $\xi(t)$ are independent.

As we all know human activities will have a significant impact on the population system, we must pay attention to the growing influence of human beings on population systems. The main manifestation of human activities is the regular harvesting of species or the regular stocking for the protection of endangered species, which cannot be considered continuously. Therefore, these phenomena can be described more accurately by the stochastic models with impulsive effects (see [29-34]).

On the other hand, human activities not only have a direct impact on the population but also have an indirect impact. The toxin produced by environmental pollution has an indirect impact on the species. Environmental pollution caused by human activities has become an important issue that the world has to consider. Environmental pollution not only pollutes the atmosphere but also produces toxins that can enter into animals and plants, causing unimaginable harm to them; the light ones can make some populations die, and the heavy ones may cause species extinction. And these toxins will also accumulate in animals and plants. People transfer toxins in their bodies by eating the animals and plants, which can cause harm to human health. Therefore, it has become an inevitable trend to consider the influence of environmental toxins on the population (see [35-38]).

Based on the above discussion, we first consider the following stochastic hybrid logistic model with two-pulse perturbations:

$$
\left\{\begin{aligned}
\mathrm{d} X(t) & \left.=X(t)\left[r(\xi(t))-\alpha(\xi(t)) C_{0}(t)-a_{1}(\xi(t)) X(t)+a_{2}(\xi(t)) X(t-\tau)+a_{3}(\xi(t)) \int_{-\infty}^{0} X(t+\theta) \mathrm{d} \varsigma(\theta)\right] \mathrm{d} t\right] \\
& +\sigma(\xi(t)) X(t) \mathrm{d} B(t)+X\left(t^{-}\right) \int_{\mathbb{V}} \gamma((\xi(t)), v) \tilde{N}(\mathrm{~d} t, \mathrm{~d} v), \\
\mathrm{d} C_{0}(t) & =\left[k C_{e}(t)-(g+m) C_{0}(t)\right] \mathrm{d} t, \\
\mathrm{~d} C_{e}(t) & =-h C_{e}(t) \mathrm{d} t, \\
\Delta X(t) & =\delta_{n} X(t), \Delta C_{0}(t)=0, \Delta C_{e}(t)=0, \quad t=t_{n}, n \in \mathbb{Z}^{+}, \\
\Delta X(t) & =0, \Delta C_{0}(t)=0, \Delta C_{e}(t)=u, \quad t=n T, n \in \mathbb{Z}^{+},
\end{aligned}\right\}, n \in \mathbb{Z}^{+},
$$

where $C_{0}(t)$ and $C_{e}(t)$ represent the concentration of toxins in organism and in environment at time $t$, respectively. $\alpha \geq 0$ is the decreasing rate of the growth rate associated with the uptake of the toxins, $k>0$ stands for the uptake rate of toxicant in the environment, $g>0$ and $m>0$ are the excretion rate and depuration rate, respectively, $h>0$ is the loss rate of toxicant in the environment, and $u$ stands for the toxin input amount at every time. Let $0<t_{1}<t_{2}<\cdots, \lim _{n \longrightarrow+\infty} t_{n}=+\infty, n \in \mathbb{Z}^{+}$, where $\mathbb{Z}^{+}$is the set of positive integers. When $\delta_{n}>0$, the impulsive effects imply releasing population, while if $\delta_{n}<0$, the impulsive effects indicate harvesting for population. In this paper, we always suppose that $1+\delta_{n}>0$ for all $n \in \mathbb{Z}^{+}$.

The rest of the paper is organized as follows. In Section 2 , we give some preliminaries. The existence and uniqueness of the global positive solution of the model are given in Section 3. The sufficient conditions for the stochastic permanence and extinction are studied in Section 4. Some asymptotic properties of the solution are proved in Section 5. Finally, we give some numerical simulations to illustrate our results.

\section{Preliminaries}

Denote the generator $\Gamma=\left(\gamma_{k l}\right)_{N \times N}$ of the Markov chain $\xi(t)$ given by

$$
\mathbb{P}(\xi(t+\Delta)=l \mid \xi(t)=k)= \begin{cases}\gamma_{k l} \Delta+o(\Delta), & \text { if } k \neq l, \\ 1+\gamma_{k k} \Delta+o(\Delta), & \text { if } k=l\end{cases}
$$

where $\Delta>0, \gamma_{k l} \geq 0$ if $k \neq l$ while $\sum_{l=1}^{N} \gamma_{k l}=0$. When $\xi(t)$ is irreducible, then $\xi(t)$ has a unique stationary distribution $\pi=\left\{\pi_{1}, \pi_{2}, \ldots, \pi_{N}\right\}$ which is the solution of 


$$
\left\{\begin{array}{l}
\pi \Gamma=0, \\
\sum_{k=1}^{N} \pi_{k}=1, \pi_{k}>0, \quad \forall k \in \mathbb{M} .
\end{array}\right.
$$

Consider a stochastic differential delay equation (SDDE) with Markovian switching and Lévy noise (see [12]) as follows:

$$
\left\{\begin{array}{l}
\mathrm{d} x(t)=f(x(t), x(t-\tau(t)), t, \xi(t)) \mathrm{d} t+g(x(t), x(t-\tau(t)), t, \xi(t)) \mathrm{d} B(t)+\int_{\mathbb{Y}} J\left(x\left(t^{-}\right), t, \xi(t), v\right) \tilde{N}(\mathrm{~d} t, \mathrm{~d} v), \\
x(0)=x_{0} \in L_{\mathscr{F}_{0}}\left([-\tau, 0] ; \mathbb{R}^{n}\right), \\
\xi(0)=\xi_{0} \in \mathbb{M}
\end{array}\right.
$$

where $x(t)=\left(x_{1}(t), x_{2}(t), \ldots, x_{n}(t)\right)^{T} \in \mathbb{R}^{n}$ is the state vector and $x(t-\tau(t))=\left(x_{1}(t-\tau(t)), x_{2}(t-\tau(t)), \ldots\right.$, $\left.x_{n}(t-\tau(t))\right)^{T} \in \mathbb{R}^{n}$ is the delayed state vector. $x\left(t^{-}\right)=$ $\lim _{s \longrightarrow t} x(s)$. The time-varying delay $\tau(\cdot): \mathbb{R}_{+} \longrightarrow[0, \tau]$ is a Borel measurable function. $f(\cdot, \cdot, \cdot, \cdot): \mathbb{R}^{n} \times \mathbb{R}^{n} \times \mathbb{R}_{+} \times$ $\mathbb{M} \longrightarrow \mathbb{R}^{n}$ is the drift coefficient vector, $g(\cdot, \cdot, \cdot, \cdot): \mathbb{R}^{n} \times \mathbb{R}^{n} \times$
$\mathbb{R}_{+} \times \mathbb{M} \longrightarrow \mathbb{R}^{n \times n}$ is the diffusion coefficient matrix, and $J(\cdot, \cdot, \cdot, \cdot): \mathbb{R}^{n} \times \mathbb{R}^{n} \times \mathbb{M} \times \mathbb{Y} \longrightarrow \mathbb{R}^{n}$.

For each $k \in \mathbb{M}$, let $V(x, y, t, k) \in C^{1,2}\left(\mathbb{R}^{n} \times \mathbb{R}^{n} \times \mathbb{R}_{+} \times\right.$ $\left.\mathbb{M} ; \mathbb{R}_{+}\right)$be any twice continuously differentiable function; the operator $\mathscr{L} V(x, y, t, k): \mathbb{R}^{n} \times \mathbb{R}^{n} \times \mathbb{R}_{+} \times \mathbb{M} \longrightarrow \mathbb{R}$ can be defined by

$$
\begin{aligned}
\mathscr{L} V(x, y, t, k)= & V_{t}(x, t, k)+V_{x}(x, t, k) f(x, y, t, k) \\
& +\frac{1}{2} \operatorname{trace}\left[g^{T}(x, y, t, k) V_{x x}(x, t, k) g(x, y, t, k)\right] \\
& +\int_{\mathbb{V}}\left[V(x+J(x, t, k, v), t, k)-V(x, t, k)-J(x, t, k, v) V_{x}(x, t, k)\right] \lambda(\mathrm{d} v)+\sum_{l=1}^{N} \gamma_{k l} V(x, t, k),
\end{aligned}
$$

where

$$
\left\{\begin{array}{l}
V_{t}(x, t, k)=\frac{\partial V(x, t, k)}{\partial t}, \\
V_{x}(x, t, k)=\left(\frac{\partial V(x, t, k)}{\partial x_{1}}, \frac{\partial V(x, t, k)}{\partial x_{2}}, \ldots, \frac{\partial V(x, t, k)}{\partial x_{n}}\right) \\
V_{x x}(x, t, k)=\left(\frac{\partial^{2} V(x, t, k)}{\partial x_{i} \partial x_{j}}\right)_{n \times n} .
\end{array}\right.
$$

Then, one has the generalized Itô's formula:

$$
\begin{aligned}
\mathrm{d} V= & \mathscr{L} V(x, y, t, k) \mathrm{d} t+V_{x}(x, t, k) f(x, y, t, k) \mathrm{d} B(t) \\
& +\int_{\mathbb{V}} \ln (1+J(x, t, k, v)) \tilde{N}(\mathrm{~d} t, \mathrm{~d} v) .
\end{aligned}
$$

Hypothesis 1 (locally Lipschitz condition). For any integer $h \geq 1$, there exists a constant $L_{k}>0$ such that

$$
\begin{aligned}
& |f(x, y, t, k)-f(\bar{x}, \bar{y}, \bar{t}, k)| \vee|g(x, y, t, k)-g(\bar{x}, \bar{t}, k)| \\
& \quad \leq L_{k}(|x-\bar{x}|+|y-\bar{y}|)
\end{aligned}
$$

for those $x, y, \bar{x}, \bar{y} \in \mathbb{R}^{n}$ with $|x| \vee|y| \vee|\bar{x}| \vee|\bar{y}| \leq h$ and any $(t, k) \in[0, T] \times \mathbb{M}$.

Hypothesis 2 (linear growth condition). There is a constant $L>0$ such that

$$
|f(x, y, t, k)| \vee|g(x, y, t, k)| \leq L(1+|x|+|y|),
$$

for any $(x, y, t, k) \in \mathbb{R}^{n} \times \mathbb{R}^{n} \times \mathbb{R}_{+} \times \mathbb{M}$.

Hypothesis 3. For each $\xi>0$, there is a constant $L_{\xi}$ that depends on $\xi$ such that

$$
\int_{\mathbb{Y}}|J(x, t, k, v)-J(y, t, k, v)|^{2} \lambda(\mathrm{d} v) \leq L_{\xi}|x-y|^{2},
$$

with $|x| \vee|y| \leq \xi$.

Here, Hypotheses 1-3 are the conservative conditions to check the existence and uniqueness of the global solution of (7). In this paper, Hypotheses 1-3 are always satisfied.

For simplicity, denote some notations 


$$
\left\{\begin{array}{l}
\langle f\rangle_{t}=\frac{1}{t} \int_{0}^{t} f(s) \mathrm{d} s, \quad t>0, \\
\widehat{g}=\min _{k \in \mathbb{M}}\{g(k)\}, \\
\check{g}=\max _{k \in \mathbb{M}}\{g(k)\} \\
f_{*}=\lim _{t \longrightarrow+\infty} f(t), \\
f^{*}=\limsup _{t \longrightarrow+\infty} f(t), \\
b(k)=r(k)-\frac{\sigma^{2}(k)}{2}-\int_{\mathbb{Y}}[\gamma((k), v)-\ln (1+\gamma((k), v))] \lambda(\mathrm{d} v), \\
\bar{b}(k)=2 r(k)-\sigma^{2}(k)-2 \int_{\mathbb{V}}(\gamma((k), v)) \lambda(\mathrm{d} v)-2 \alpha(k) C_{0}^{*}, \\
\left.\eta^{*}=\limsup _{t \longrightarrow+\infty} \frac{1}{t}\left[\sum_{0<t_{n}<t} \ln \left(1+\delta_{n}\right)\right]+\sum_{k \in \mathbb{M}} \pi_{k} b(k), \eta_{*}=\lim _{t \longrightarrow+\infty} \frac{1}{t} \sum_{0<t_{n}<t} \ln \left(1+\delta_{n}\right)\right]+\sum_{k \in \mathbb{M}} \pi_{k} b(k) .
\end{array}\right.
$$

In order to give the proof in this paper, we provide some assumptions.

A.1: there exists a constant $K_{1}>0$ such that

$$
\int_{\mathbb{Y}} \ln (1+\gamma((\xi(t)), v))^{2} \lambda(\mathrm{d} v) \leq K_{1} .
$$

A.2: $1+\gamma((\xi(t)), v)>0$, and there exists constant $K_{2}>0$ such that

$$
\int_{\mathbb{V}}[\gamma((\xi(t)), v)-\ln (1+\gamma((\xi(t)), v))] \lambda(\mathrm{d} v)<K_{2} .
$$

A.3: let the initial value $X=\beta$ be positive and $\beta \in C_{g}$ (see $[31,39]$ ), which is defined by

$C_{g}=\left\{\psi \in C\left((-\infty, 0] ; \mathbb{R}_{+}\right):\|\psi\|_{C_{g}}=\sup _{-\infty<\theta \leq 0} e^{r \theta}|\varphi(\theta)|<+\infty\right\}$.

There exists a probability measure $\rho$ and a constant $r>0$ such that

$$
\rho_{r}=\int_{-\infty}^{0} e^{-2 r \theta} \mathrm{d} \varsigma(\theta)<+\infty .
$$

A.4: $a=\left(\widehat{a}_{1}-\check{a}_{2}-\check{a}_{3}\right)>0$.

A.5: there exist two positive constants $m$ and $M$ such that

$$
m \leq \prod_{0<t_{n}<t}\left(1+\delta_{n}\right) \leq M .
$$

$$
\text { A.6: } m \widehat{a}_{1}>\max \left\{M\left(\check{a}_{2}+\check{a}_{3}\right), M\left(\check{a}_{2} e^{\tau}+\check{a}_{3} \rho_{r}\right)\right\} \text {. }
$$

We give some useful inequality in [40].

(1) (Exponential martingale inequality) Let $T, \alpha, \beta$ be any positive numbers. Then,

$$
\mathbb{P}\left\{\sup _{0 \leq t \leq T}\left[\int_{0}^{t} g(s) \mathrm{d} B(s)-\frac{\alpha}{2} \int_{0}^{t}|g(s)|^{2} \mathrm{~d} s\right]>\beta\right\} \leq e^{-\alpha \beta} .
$$

(2) (Chebyshev's inequality) If $c>0, \quad p>0, X \in L^{p}$ $\left(\Omega, \mathbb{R}^{d}\right)$ which is the family of $\mathbb{R}^{d}$-valued random variables $X$ with $\mathbb{E}|X|^{p}<\infty$. Then,

$$
\mathbb{P}\{\omega:|X(\omega)| \geq c\} \leq c^{-p} \mathbb{E}|X|^{p} .
$$

Next, we consider the following subsystem of system (4):

$$
\left\{\begin{array}{l}
\mathrm{d} C_{0}(t)=\left[k C_{e}(t)-(g+m) C_{0}(t)\right] \mathrm{d} t, \\
\mathrm{~d} C_{e}(t)=-h C_{e}(t) \mathrm{d} t, \\
\Delta C_{0}(t)=0, \Delta C_{e}(t)=u, t=n T, \quad n \in n T, n \in \mathbb{Z}^{+},
\end{array}\right.
$$

From [38], we can get the following lemma.

Lemma 1 (see [38]). System (22) has a unique globally asymptotical stable positive T-periodic solution $\left(\widetilde{C}_{0}(t), \widetilde{C}_{e}(t)\right)^{T}$. If $C_{0}(0)>\widetilde{C}_{0}(0), \quad C_{e}(0)>\widetilde{C}_{e}(0)$, then $C_{0}(t)>\widetilde{C}_{0}(t), C_{e}(t)>\widetilde{C}_{e}(t)$ for all $t \geq 0$, where 


$$
\left\{\begin{array}{l}
\widetilde{C}_{0}(t)=\widetilde{C}_{0}(0) e^{-(g+m)(t-n T)}+\frac{k u\left(e^{-(g+m)(t-n T)}-e^{-h(t-n T)}\right)}{(h-g-m)\left(1-e^{-h T}\right)} \\
\widetilde{C}_{0}(0)=\frac{k u\left(e^{-(g+m) T}-e^{-h T}\right)}{(h-g-m)\left(1-e^{-(g+m) T}\right)\left(1-e^{-h T}\right)} \\
\widetilde{C}_{e}(t)=\frac{u e^{-h(t-n T)}}{1-e^{-h T}} \\
\widetilde{C}_{e}(0)=\frac{u}{1-e^{-h T}},
\end{array}\right.
$$

for $t \in(n T,(n+1) T], n \in \mathbb{Z}^{+}$. In addition,

$$
\lim _{t \longrightarrow+\infty}\left\langle C_{0}(t)\right\rangle=\frac{k u}{h(g+m) T} \triangleq \bar{C}_{0} .
$$

From Lemma 1, system (4) can be replaced by the dynamical behaviors of the following limiting system:

$$
\left\{\begin{array}{l}
\mathrm{d} X(t)=X(t)\left[r(\xi(t))-\alpha(\xi(t)) C_{0}(t)-a_{1}(\xi(t)) X(t)+a_{2}(\xi(t)) X(t-\tau)+a_{3}(\xi(t)) \int_{-\infty}^{0} X(t+\theta) \mathrm{d} \varsigma(\theta)\right] \mathrm{d} t \\
+\sigma(\xi(t)) X(t) \mathrm{d} B(t)+x\left(t^{-}\right) \int_{\mathbb{V}} \gamma((\xi(t)), v) \tilde{N}(\mathrm{~d} t, \mathrm{~d} v), \\
\Delta X(t)=\delta_{n} X(t), t=t_{n}, \quad n \in \mathbb{Z}^{+} .
\end{array}\right\}, \quad t \neq t_{n}, n \in \mathbb{Z}^{+},
$$

\section{Positive and Global Solutions}

Theorem 1. For any initial data $(X(0), \xi(0)) \in \mathbb{R} \times \mathbb{M}$, system (25) has a unique positive solution $(X(t), \xi(t)) \in \mathbb{R} \times$ $\mathbb{M}$ with probability one
Proof. Consider the following SDDEs with Markovian switching and without impulses:

$$
\begin{aligned}
\mathrm{d} Y(t)= & Y(t)\left[r(\xi(t))-\alpha(\xi(t)) C_{0}(t)-a_{1}(\xi(t)) \prod_{0<t_{n}<t}\left(1+\delta_{n}\right) Y(t)\right. \\
& +a_{2}(\xi(t)) \prod_{0<t_{n}<t-\tau}\left(1+\delta_{n}\right) Y(t-\tau) \\
& \left.+a_{3}(\xi(t)) \int_{-\infty}^{0} \prod_{0<t_{n}<t+\theta}\left(1+\delta_{n}\right) Y(t+\theta) \mathrm{d} \varsigma(\theta)\right] \mathrm{d} t \\
& +\sigma(\xi(t)) Y(t) \mathrm{d} B(t)+Y\left(t^{-}\right) \int_{\mho} \gamma((\xi(t)), v) \tilde{N}(\mathrm{~d} t, \mathrm{~d} v)
\end{aligned}
$$

with initial value $(Y(0), \xi(0))$. By the theory of SDDEs with Markovian switching and Lévy jump, we refer the reader to [12]. System (26) has a unique global positive solution $(Y(t), \xi(t))$.
Let $X(t)=\prod_{0<t_{n}<t}\left(1+\delta_{n}\right) Y(t)$ with initial value $(X(0), \xi(0))$.

Since $X(t)$ is continuous on each interval $\left(t_{n}, t_{n+1}\right) \subset \mathbb{R}_{+}$, then

$$
\begin{aligned}
\mathrm{d} X(t)= & \prod_{0<t_{n}<t}\left(1+\delta_{n}\right) \mathrm{d} Y(t) \\
= & \prod_{0<t_{n}<t}\left(1+\delta_{n}\right) Y(t)\left[r(\xi(t))-\alpha(\xi(t)) C_{0}(t)-a_{1}(\xi(t)) \prod_{0<t_{n}<t}\left(1+\delta_{n}\right) Y(t)\right. \\
& \left.+a_{2}(\xi(t)) \prod_{0<t_{n}<t-\tau}\left(1+\delta_{n}\right) Y(t-\tau)+a_{3}(\xi(t)) \int_{-\infty}^{0} \prod_{0<t_{n}<t+\theta}\left(1+\delta_{n}\right) Y(t+\theta) \mathrm{d} \varsigma(\theta)\right] \mathrm{d} t
\end{aligned}
$$




$$
\begin{aligned}
& +\sigma(\xi(t)) \prod_{0<t_{n}<t}\left(1+\delta_{n}\right) Y(t) \mathrm{d} B(t)+\prod_{0<t_{n}<t}\left(1+\delta_{n}\right) Y\left(t^{-}\right) \int_{\mathbb{V}} \gamma((\xi(t)), v) \tilde{N}(\mathrm{~d} t, \mathrm{~d} v) \\
= & X(t)\left[r(\xi(t))-\alpha(\xi(t)) C_{0}(t)-a_{1}(\xi(t)) X(t)+a_{2}(\xi(t)) X(t-\tau)\right. \\
& \left.+a_{3}(\xi(t)) \int_{-\infty}^{0} X(t+\theta) \mathrm{d} \varsigma(\theta)\right] \mathrm{d} t+\sigma(\xi(t)) X(t) \mathrm{d} B(t)+X\left(t^{-}\right) \int_{\mathbb{V}} \gamma((\xi(t)), v) \tilde{N}(\mathrm{~d} t, \mathrm{~d} v),
\end{aligned}
$$

for $t \neq t_{n}, n \in \mathbb{Z}^{+}$.

And for $\forall t_{n} \in \mathbb{R}_{+}$, we get

$$
\begin{aligned}
X\left(t_{n}^{+}\right) & =\lim _{t \longrightarrow t_{n}^{+}} X(t)=\prod_{0<t_{n}<t}\left(1+\delta_{n}\right) Y\left(t_{n}^{+}\right) \\
& =\left(1+\delta_{n}\right) \prod_{0<t_{n}<t}\left(1+\delta_{n}\right) Y\left(t_{n}\right)=\left(1+\delta_{n}\right) X\left(t_{n}\right) .
\end{aligned}
$$

Moreover,

$$
\begin{aligned}
X\left(t_{n}^{-}\right) & =\lim _{t \longrightarrow t_{n}^{-}} X(t)=\prod_{0<t_{n}<t}\left(1+\delta_{n}\right) Y\left(t_{n}^{-}\right) \\
& =\prod_{0<t_{n}<t}\left(1+\delta_{n}\right) Y\left(t_{n}\right)=X\left(t_{n}\right) .
\end{aligned}
$$

Thus, system (25) has the unique global positive solution $(X(t), \xi(t))$.

\section{Extinction and Persistence}

Theorem 2. When A.1-A.4 hold, if $\eta^{*}<\widehat{\alpha} \bar{C}_{0}$, then

$$
\lim _{t \longrightarrow+\infty} X(t)=0, \quad \text { a.s. }
$$

Namely, the population $X(t)$ of system (25) is extinct.

Proof. Applying Itô's formula to system (26), we have

$$
\begin{aligned}
\mathrm{d} \ln Y(t)= & {\left[r(\xi(t))-\frac{\sigma^{2}(\xi(t))}{2}-\int_{\mathbb{Y}}[\gamma((\xi(t)), v)-\ln (1+\gamma((\xi(t)), v))] \lambda(\mathrm{d} v)\right.} \\
& -\alpha(\xi(t)) C_{0}(t)-a_{1}(\xi(t)) \prod_{0<t_{n}<t}\left(1+\delta_{n}\right) Y(t) \\
& +a_{2}(\xi(t)) \prod_{0<t_{n}<t-\tau}\left(1+\delta_{n}\right) Y(t-\tau) \\
& \left.+a_{3}(\xi(t)) \int_{-\infty}^{0} \prod_{0<t_{n}<t+\theta}\left(1+\delta_{n}\right) Y(t+\theta) \mathrm{d} \xi(\theta)\right] \mathrm{d} t \\
& +\sigma(\xi(t)) \mathrm{d} B(t)+\int_{\mathbb{Y}} \ln (1+\gamma((\xi(t)), v)) \tilde{N}(\mathrm{~d} t, \mathrm{~d} v)
\end{aligned}
$$

Integrating both sides of (31) from 0 to $t$ yields

$$
\begin{aligned}
\ln Y(t)-\ln Y(0)= & \int_{0}^{t}\left[b(\xi(s))-\alpha(\xi(s)) C_{0}(s)-a_{1}(\xi(s)) \prod_{0<t_{n}<s}\left(1+\delta_{n}\right) Y(s)\right. \\
& +a_{2}(\xi(s)) \prod_{0<t_{n}<s-\tau}\left(1+\delta_{n}\right) Y(s-\tau) \\
& \left.+a_{3}(\xi(s)) \int_{-\infty}^{0} \prod_{0<t_{n}<s+\theta}\left(1+\delta_{n}\right) Y(s+\theta) \mathrm{d} \xi(\theta)\right] \mathrm{d} s \\
& +\sigma(\xi(s)) \mathrm{d} B(s)+\int_{\mathbb{V}} \ln (1+\gamma((\xi(s)), v)) \tilde{N}(\mathrm{~d} s, \mathrm{~d} v)
\end{aligned}
$$




$$
\begin{aligned}
& =\int_{0}^{t}\left[b(\xi(s))-\alpha(\xi(s)) C_{0}(s)-a_{1}(\xi(s)) X(s)+a_{2}(\xi(s)) X(s-\tau)\right. \\
& \left.+a_{3}(\xi(s)) \int_{-\infty}^{0} X(s+\theta) \mathrm{d} \xi(\theta)\right] \mathrm{d} s+\int_{0}^{t} \sigma(\xi(s)) \mathrm{d} B(s) \\
& \quad+\int_{0}^{t} \int_{\mho} \ln (1+\gamma((\xi(s)), v)) \tilde{N}(\mathrm{~d} s, \mathrm{~d} v),
\end{aligned}
$$

owing to

$$
\begin{aligned}
\int_{0}^{t} a_{3}(\xi(s)) \int_{-\infty}^{0} X(s+\theta) \mathrm{d} \xi(\theta) \mathrm{d} s= & \int_{0}^{t} a_{3}(\xi(s))\left[\int_{-\infty}^{-s} X(s+\theta) \mathrm{d} \xi(\theta)+\int_{-s}^{0} X(s+\theta) \mathrm{d} \xi(\theta)\right] \mathrm{d} s \\
= & \int_{0}^{t} a_{3}(\xi(s)) \mathrm{d} s \int_{-\infty}^{-s} e^{r(s+\theta)} X(s+\theta) e^{-r(s+\theta)} \mathrm{d} \xi(\theta) \\
& +\int_{-t}^{0} \mathrm{~d} \xi(\theta) \int_{-\theta}^{t} a_{3}(\xi(s)) X(s+\theta) \mathrm{d} s \\
\leq & \check{a}_{3}\|\varphi\|_{C_{g}} \int_{0}^{t} e^{-r s} \mathrm{~d} s \int_{-\infty}^{0} e^{-2 r \theta} \mathrm{d} \xi(\theta)+\check{a}_{3} \int_{-\infty}^{0} \mathrm{~d} \xi(\theta) \int_{0}^{t} X(s) \mathrm{d} s \\
\leq & \frac{1}{r} \check{a}_{3}\|\varphi\|_{C_{g}} \rho_{r}\left(1-e^{-r t}\right)+\check{a}_{3} \int_{0}^{t} X(s) \mathrm{d} s .
\end{aligned}
$$

Then, we have

$$
\begin{aligned}
\ln Y(t)-\ln Y(0)= & \int_{0}^{t}\left[b(\xi(s))-\alpha(\xi(s)) C_{0}(s)-a_{1}(\xi(s)) X(s)+a_{2}(\xi(s)) X(s-\tau)\right. \\
& \left.+a_{3}(\xi(s)) \int_{-\infty}^{0} X(s+\theta) \mathrm{d} \xi(\theta)\right] \mathrm{d} s+\int_{0}^{t} \sigma(\xi(s)) \mathrm{d} B(s) \\
& +\int_{0}^{t} \int_{\mathbb{V}} \ln (1+\gamma((\xi(s)), v)) \tilde{N}(\mathrm{~d} s, \mathrm{~d} v) \\
\leq & \int_{0}^{t}\left[b(\xi(s))-\alpha(\xi(s)) C_{0}(s)-\left(\widehat{a}_{1}-\check{a}_{2}-\check{a}_{3}\right) X(s)\right] \mathrm{d} s \\
& +\frac{1}{r} \check{a}_{3}\|\varphi\|_{C_{g}} \rho_{r}\left(1-e^{-r t}\right)+\int_{-\tau}^{0} a_{2}(\xi(s+\tau)) X(s) \mathrm{d} s \\
& +\int_{0}^{t} \sigma(\xi(s)) \mathrm{d} B(s)+\int_{0}^{t} \int_{\mathbb{V}} \ln (1+\gamma((\xi(s)), v)) \widetilde{N}(\mathrm{~d} s, \mathrm{~d} v) \\
\leq & \int_{0}^{t}\left[b(\xi(s))-\alpha(\xi(t)) C_{0}(s)-a X(s)\right] \mathrm{d} s+\frac{1}{r} \check{a}_{3}\|\varphi\|_{C_{g}} \rho_{r}\left(1-e^{-r t}\right) \\
& +\int_{-\tau}^{0} a_{2}(\xi(s+\tau)) X(s) \mathrm{d} s+M_{1}(t)+M_{2}(t),
\end{aligned}
$$


where

$$
\begin{aligned}
& M_{1}(t)=\int_{0}^{t} \sigma(\xi(s)) \mathrm{d} B(s), \\
& M_{2}(t)=\int_{0}^{t} \int_{\mathbb{Y}} \ln (1+\gamma((\xi(s)), v)) \tilde{N}(\mathrm{~d} s, \mathrm{~d} v) .
\end{aligned}
$$

Since $M_{1}(t)$ and $M_{2}(t)$ are local martingales, the quadratic variations are

$$
\begin{aligned}
& \left\langle M_{1}(t), M_{1}(t)\right\rangle=\int_{0}^{t} \sigma^{2}(\xi(s)) \mathrm{d} s \leq\left(\check{\sigma}_{1}\right)^{2} t, \\
& \left\langle M_{2}(t), M_{2}(t)\right\rangle=\int_{0}^{t} \int_{\Psi} \ln (1+\gamma((\xi(s)), v))^{2} \lambda(\mathrm{d} v) \mathrm{d} s \leq K_{1} t .
\end{aligned}
$$

Making use of the strong law of large numbers for local martingales (see [41]) yields

$$
\begin{aligned}
& \lim _{t \rightarrow+\infty} \frac{M_{1}(t)}{t}=0, \\
& \lim _{t \rightarrow+\infty} \frac{M_{2}(t)}{t}=0,
\end{aligned}
$$

a.s.

From (34), we can get that

$$
\begin{aligned}
\sum_{0<t_{n}<t} \ln \left(1+\delta_{n}\right)+\ln Y(t) \leq & \sum_{0<t_{n}<t} \ln \left(1+\delta_{n}\right)+\int_{0}^{t} b(\xi(s)) \mathrm{d} s-\int_{0}^{t} \alpha(\xi(t)) C_{0}(s) \mathrm{d} s \\
& -\int_{0}^{t} a X(s) \mathrm{d} s+\frac{1}{r} \check{a}_{3}\|\varphi\|_{C_{g}} \rho_{r}\left(1-e^{-r t}\right) \\
& +\int_{-\tau}^{0} a_{2}(\xi(s+\tau)) X(s) \mathrm{d} s+\ln Y(0)+M_{1}(t)+M_{2}(t) .
\end{aligned}
$$

Thus,

$$
\begin{aligned}
\ln X(t) \leq & \sum_{0<t_{n}<t} \ln \left(1+\delta_{n}\right)+\int_{0}^{t} b(\xi(s)) \mathrm{d} s-\int_{0}^{t} \alpha(\xi(t)) C_{0}(s) \mathrm{d} s \\
& -\int_{0}^{t} a X(s) \mathrm{d} s+\frac{1}{r} \check{a}_{3}\|\varphi\|_{C_{g}} \rho_{r}\left(1-e^{-r t}\right) \\
& +\int_{-\tau}^{0} a_{2}(\xi(s+\tau)) X(s) \mathrm{d} s+\ln Y(0)+M_{1}(t)+M_{2}(t) \\
\leq & \sum_{0<t_{n}<t} \ln \left(1+\delta_{n}\right)+\int_{0}^{t} b(\xi(s)) \mathrm{d} s-\widehat{\alpha} \int_{0}^{t} C_{0}(s) \mathrm{d} s \\
& -a \int_{0}^{t} X(s) \mathrm{d} s+\frac{1}{r} \check{a}_{3}\|\varphi\|_{C_{g}} \rho_{r}\left(1-e^{-r t}\right) \\
& +\int_{-\tau}^{0} a_{2}(\xi(s+\tau)) X(s) \mathrm{d} s+\ln Y(0)+M_{1}(t)+M_{2}(t) .
\end{aligned}
$$

Taking superior limit on both sides of (39) and applying the ergodicity of $\xi(\cdot)$ and (37), we obtain

$$
\begin{aligned}
\limsup _{t \longrightarrow+\infty} \frac{\ln X(t)}{t} \leq & \limsup _{t \longrightarrow+\infty} \frac{1}{t}\left[\sum_{0<t_{n}<t} \ln \left(1+\delta_{n}\right)\right] \\
& +\sum_{k \in \mathbb{M}} \pi_{k} b(k)-\widehat{\alpha} \bar{C}_{0}:=\eta^{*}-\widehat{\alpha} \bar{C}_{0}<0, \quad \text { a.s. }
\end{aligned}
$$

Theorem 3. When A.1-A.4 hold, if $\eta^{*} \geq \widehat{\alpha} \bar{C}_{0}$, then

$$
\underset{t \longrightarrow+\infty}{\limsup } \frac{1}{t} \int_{0}^{t} X(s) \mathrm{d} s \leq \frac{\eta^{*}-\widehat{\alpha} \bar{C}_{0}}{a}=\bar{X}^{*}
$$

Particularly, if $\eta^{*}=\widehat{\alpha} \bar{C}_{0}$, then $\lim _{t \rightarrow+\infty}(1 / t)$ $\int_{0}^{t} X(s) d s=0$, that is, the population $X(t)$ of system (25) is nompersistent in the mean.

Proof. For $\forall \varepsilon>0$, there exists a constant $T>0$, for all $0<T<n-1 \leq t \leq n$, such that 


$$
\begin{aligned}
& \frac{1}{t}\left[\ln Y(0)+\frac{1}{r} \check{a}_{3}\|\varphi\|_{C_{g}} \rho_{r}\left(1-e^{-r t}\right)+\int_{-\tau}^{0} a_{2}(\xi(s+\tau)) X(s) \mathrm{d} s+M_{1}(t)+M_{2}(t)\right] \leq \frac{\varepsilon}{2}, \\
& \quad \frac{1}{t}\left[\sum_{0<t_{n}<t} \ln \left(1+\delta_{n}\right)+\int_{0}^{t} b(\xi(s)) \mathrm{d} s-\widehat{\alpha} \int_{0}^{t} C_{0}(s) \mathrm{d} s\right] \leq \eta^{*}-\widehat{\alpha} \bar{C}_{0}+\frac{\varepsilon}{2} .
\end{aligned}
$$

Substituting above inequalities into (39), we have

$$
\begin{aligned}
\ln X(t) \leq & \sum_{0<t_{n}<t} \ln \left(1+\delta_{n}\right)+\int_{0}^{t} b(\xi(s)) \mathrm{d} s-\widehat{\alpha} \int_{0}^{t} C_{0}(s) \mathrm{d} s \\
& -a \int_{0}^{t} X(s) \mathrm{d} s+\frac{1}{r} \check{a}_{3}\|\varphi\|_{C_{g}} \rho_{r}\left(1-e^{-r t}\right) \\
& +\int_{-\tau}^{0} a_{2}(\xi(s+\tau)) X(s) \mathrm{d} s+\ln Y(0)+M_{1}(t)+M_{2}(t) \\
\leq & \left(\eta^{*}-\widehat{\alpha} \bar{C}_{0}+\varepsilon\right) t-a \int_{0}^{t} X(s) \mathrm{d} s, \quad \text { a.s. }
\end{aligned}
$$

Denote $g(t)=\int_{0}^{t} X(s) \mathrm{d} s$; then, we have $(\mathrm{d} g(t) / \mathrm{d} t)=$ $X(t)$. Taking exponent on both sides of (43) yields

$$
e^{a g(t)} \frac{\mathrm{d} g(t)}{\mathrm{d} t} \leq e^{\left(\eta^{*}-\widehat{\alpha}_{0}+\varepsilon\right) t}
$$

Integrating (44) from $T$ to $t$, we can show that

$$
\begin{aligned}
e^{a g(t)} \leq & \frac{a}{\eta^{*}-\widehat{\alpha} \bar{C}_{0}+\varepsilon} e^{\left(\eta^{*}-\widehat{\alpha C}_{0}+\varepsilon\right) t}+e^{a g\left(T_{0}\right)} \\
& -\frac{a}{\eta^{*}-\widehat{\alpha} \bar{C}_{0}+\varepsilon} e^{\left(\eta^{*}-\widehat{\alpha} \bar{C}_{0}+\varepsilon\right) T} .
\end{aligned}
$$

Taking logarithm of (45) yields

$$
\begin{aligned}
\int_{0}^{t} X(s) \mathrm{d} s \leq & \frac{1}{a} \ln \left[\frac{a}{\eta^{*}-\widehat{\alpha} \bar{C}_{0}+\varepsilon} e^{\left(\eta^{*}-\widehat{\alpha} \bar{C}_{0}+\varepsilon\right) t}\right. \\
& \left.+e^{a g(T)}-\frac{a}{\eta^{*}-\widehat{\alpha} \bar{C}_{0}+\varepsilon} e^{\left(\eta^{*}-\widehat{\alpha C}_{0}+\varepsilon\right) T}\right] .
\end{aligned}
$$

Taking superior limit on (46) elicits that

$$
\begin{aligned}
\limsup _{t \longrightarrow+\infty} \frac{1}{t} \int_{0}^{t} X(s) \mathrm{d} s \leq & \limsup _{t \longrightarrow+\infty} \frac{1}{a t} \ln \left(\frac{a}{\eta^{*}-\widehat{\alpha} \bar{C}_{0}+\varepsilon} e^{\left(\eta^{*}-\widehat{\alpha} \bar{C}_{0}+\varepsilon\right) t}\right. \\
& \left.+e^{a g(T)}-\frac{a}{\eta^{*}-\widehat{\alpha} \bar{C}_{0}+\varepsilon} e^{\left(\eta^{*}-\widehat{\alpha} \bar{C}_{0}+\varepsilon\right) T}\right) .
\end{aligned}
$$

Utilizing L'Hospital's rule results in

$$
\underset{t \longrightarrow+\infty}{\limsup } \frac{1}{t} \int_{0}^{t} X(s) \mathrm{d} s \leq \limsup _{t \longrightarrow+\infty} \frac{\eta^{*}-\widehat{\alpha} \bar{C}_{0}+\varepsilon}{a}=\frac{\eta^{*}-\widehat{\alpha} \bar{C}_{0}}{a}=\bar{X}^{*} \text {. }
$$

Theorem 4. When A.1-A.4 hold, if $\eta^{*}>\widehat{\alpha} \bar{C}_{0}$, then the population $X(t)$ of system (25) is weakly persistent a.s.

Proof. Denote $L=\left\{\omega \mid \limsup _{t \longrightarrow+\infty} X(t, \omega)=0\right\}$; suppose that $\mathbb{P}(L)>0$. Then, it follows from (39) that

$$
\begin{aligned}
\frac{\ln X(t)-\ln X(0)}{t} \leq & \frac{1}{t}\left[\sum_{0<t_{n}<t} \ln \left(1+\delta_{n}\right)+\int_{0}^{t} b(\xi(s)) \mathrm{d} s-\widehat{\alpha} \int_{0}^{t} C_{0}(s) \mathrm{d} s\right] \\
& -\frac{a}{t} \int_{0}^{t} X(s) \mathrm{d} s+\frac{1}{r t} \check{a}_{3}\|\varphi\|_{C_{g}} \rho_{r}\left(1-e^{-r t}\right) \\
& +\frac{1}{t} \int_{-\tau}^{0} a_{2}(\xi(s+\tau)) X(s) \mathrm{d} s+\frac{M_{1}(t)}{t}+\frac{M_{2}(t)}{t} .
\end{aligned}
$$

For $\forall \omega \in L$, we have $\lim _{t \longrightarrow+\infty} X(t, \omega)=0$. As a result,

$$
\begin{aligned}
\limsup _{t \longrightarrow+\infty} & \frac{\ln X(t, \omega)-\ln X(0)}{t} \leq 0, \\
\limsup _{t \longrightarrow+\infty} \frac{a}{t} \int_{0}^{t} X(s) \mathrm{d} s & =0 .
\end{aligned}
$$

From (49), one has

$$
0 \geq \limsup _{t \rightarrow+\infty} \ln X(t, w)=\eta^{*}-\widehat{\alpha} \bar{C}_{0}>0,
$$

which is a contradiction.

Remark 1. Through Theorems 2-4, we find an interesting biological phenomenon: when $\eta^{*}>\widehat{\alpha} \bar{C}_{0}$, the population $X(t)$ is weakly persistent; when $\eta^{*}<\widehat{\alpha} \bar{C}_{0}$, the population $X(t)$ goes to extinction, which means that the persistence and extinction of $X(t)$ depend on $\eta^{*}$ and the absorption intensity of toxins $\widehat{\alpha} \bar{C}_{0}$.

Theorem 5. When A.1-A.3 hold, if $\eta_{*}>\check{\alpha} \bar{C}_{0}$, then 


$$
\liminf _{t \longrightarrow+\infty} \frac{1}{t} \int_{0}^{t} X(s) \mathrm{d} s \geq \frac{\eta_{*}-\check{\alpha} \bar{C}_{0}}{\check{a}_{1}}=\underline{X}, \quad \text { a.s. }
$$

That is, the population $X(t)$ of system (25) is persistent in the mean a.s.

Proof. Applying Itô's formula to (26) yields

$$
\begin{aligned}
\mathrm{d} \ln Y(t)= & {\left[r(\xi(t))-\frac{\sigma^{2}(\xi(t))}{2}-\int_{\mathbb{V}}[\gamma((\xi(t)), v)-\ln (1+\gamma((\xi(t)), v))] \lambda(\mathrm{d} v)\right.} \\
& -\alpha(\xi(t)) C_{0}(t)-a_{1}(\xi(t)) \prod_{0<t_{n}<t}\left(1+\delta_{n}\right) Y(t) \\
& +a_{2}(\xi(t)) \prod_{0<t_{n}<t-\tau}\left(1+\delta_{n}\right) Y(t-\tau) \\
& \left.+a_{3}(\xi(t)) \int_{-\infty}^{0} \prod_{0<t_{n}<t+\theta}\left(1+\delta_{n}\right) Y(t+\theta) \mathrm{d} \xi(\theta)\right] \mathrm{d} t \\
& +\sigma(\xi(t)) \mathrm{d} B(t)+\int_{\mathbb{V}} \ln (1+\gamma((\xi(t)), v)) \tilde{N}(\mathrm{~d} t, \mathrm{~d} v) \\
\geq & {\left[b(\xi(t))-\check{\alpha} C_{0}(t)-\check{a}_{1} X(t)\right] \mathrm{d} t+\sigma(\xi(t)) \mathrm{d} B(t) } \\
& +\int_{\mathbb{Y}} \ln (1+\gamma((\xi(t)), v)) \tilde{N}(\mathrm{~d} t, \mathrm{~d} v) .
\end{aligned}
$$

Calculating inequality (53), one has

$$
\begin{aligned}
\ln X(t) \geq & \sum_{0<t_{n}<t} \ln \left(1+\delta_{n}\right)+\int_{0}^{t} b(\xi(s)) \mathrm{d} s-\check{\alpha} \int_{0}^{t} C_{0}(s) \mathrm{d} s \\
& -\check{a}_{1} \int_{0}^{t} X(s) \mathrm{d} s+\ln Y(0)+M_{1}(t)+M_{2}(t) .
\end{aligned}
$$

According to the properties of the limit, there exists $T_{1}>0$ such that for $\forall \varepsilon_{1}>0$,

$$
\begin{aligned}
\sum_{0<t_{n}<t} \ln \left(1+\delta_{n}\right)+\int_{0}^{t} b(\xi(s)) \mathrm{d} s-\check{\alpha} \int_{0}^{t} C_{0}(s) \mathrm{d} s & \geq\left(\eta_{*}-\check{\alpha} \bar{C}_{0}-\frac{\varepsilon_{1}}{4}\right) t, \\
\ln y(0) & \geq-\frac{\varepsilon_{1}}{4} t, \\
M_{1}(t) & \geq-\frac{\varepsilon_{1}}{4} t \\
M_{2}(t) & \geq-\frac{\varepsilon_{1}}{4} t .
\end{aligned}
$$

Then, inequality (54) becomes

$$
\ln X(t) \geq\left(\eta_{*}-\check{\alpha} \bar{C}_{0}-\varepsilon_{1}\right) t-\check{a}_{1} \int_{0}^{t} X(s) \mathrm{d} s,
$$

for $t>T_{1}$. By using a method similar to Theorem 3, we can obtain that

$$
\liminf _{t \longrightarrow+\infty} \frac{1}{t} \int_{0}^{t} X(s) \mathrm{d} s \geq \liminf _{t \longrightarrow+\infty} \frac{\eta_{*}-\check{\alpha} \bar{C}_{0}-\varepsilon_{1}}{\check{a}_{1}}=\frac{\eta_{*}-\check{\alpha} \bar{C}_{0}}{\check{a}_{1}}=\stackrel{*}{X} .
$$

Theorem 6. When A.1-A.3, A.5, and A.6 hold, if $\sum_{k=1}^{N} \pi_{k} \bar{b}(k)>0$, then the population $X(t)$ of system (25) is stochastically permanent.

Proof. First, we prove that for $\forall \varepsilon>0$, there exists a constant $b_{1}>0$ such that

$$
\liminf _{t \longrightarrow+\infty} \mathbb{P}\left\{X(t) \geq b_{1}\right\} \geq 1-\varepsilon
$$

Define

$$
\begin{aligned}
& V_{1}(Y)=\frac{1}{Y^{2}} \\
& V_{2}(Y)=\vartheta_{k}\left(1+V_{1}(Y)\right)^{\varrho}, \\
& V_{3}(Y)=e^{\kappa t} V_{2}(Y),
\end{aligned}
$$

where $\varrho$ and $\kappa$ are sufficiently small positive constants and satisfy

$$
\begin{aligned}
& \vartheta_{k}\left(\varrho \bar{b}(k)-2 \varrho^{2} \sigma^{2}(k)\right)-\sum_{l=1}^{N} \gamma_{k l} \vartheta_{l}>0, \quad 1 \leq k \leq N, \\
& \vartheta_{k}\left(\varrho \bar{b}(k)-2 \varrho^{2} \sigma^{2}(k)\right)-\sum_{l=1}^{N} \gamma_{k l} \vartheta_{l}-\kappa \vartheta_{k}>0, \quad 1 \leq k \leq N,
\end{aligned}
$$

where $\vartheta=\left(\vartheta_{1}, \vartheta_{2}, \ldots, \vartheta_{N}\right)^{T} \gg 0$ such that $A(\varrho) \vartheta \gg 0$.

From (26), we can calculate that 


$$
\begin{aligned}
\mathrm{d} V_{1}(Y)= & 2 V_{1}(Y)\left[-r(\xi(t))+\frac{3}{2} \sigma^{2}(\xi(t))+\alpha(\xi(t)) C_{0}(t)+a_{1}(\xi(t)) \prod_{0<t_{n}<t}\left(1+\delta_{n}\right) Y(t)\right. \\
& -a_{2}(\xi(t)) \prod_{0<t_{n}<t-\tau}\left(1+\delta_{n}\right) Y(t-\tau)-a_{3}(\xi(t)) \int_{-\infty}^{0} \prod_{0<t_{n}<t+\theta}\left(1+\delta_{n}\right) Y(t+\theta) \mathrm{d} c(\theta) \\
& \left.+\frac{1}{2} \int_{\mathbb{V}}\left(\frac{1}{(1+\gamma((\xi(t)), v))^{2}}-1+2 \gamma((\xi(t)), v)\right) \lambda(\mathrm{d} v)\right] \mathrm{d} t \\
& -2 V_{1}(Y) \sigma(\xi(t)) \mathrm{d} B(t)+V_{1}(Y) \int_{\mathbb{V}}\left(\frac{1}{(1+\gamma((\xi(t)), v))^{2}}-1\right) \tilde{N}(\mathrm{~d} t, \mathrm{~d} v) \\
\leq & 2 V_{1}(Y)\left[-r(\xi(t))+\frac{3}{2} \sigma^{2}(\xi(t))+\alpha(\xi(t)) C_{0}(t)+a_{1}(\xi(t)) \prod_{0<t_{n}<t}\left(1+\delta_{n}\right) Y(t)\right. \\
& \left.+\frac{1}{2} \int_{\mathbb{V}}\left(\frac{1}{(1+\gamma((\xi(t)), v))^{2}}-1+2 \gamma((\xi(t)), v)\right) \lambda(\mathrm{d} v)\right] \mathrm{d} t \\
& -2 V_{1}(Y) \sigma(\xi(t)) \mathrm{d} B(t)+V_{1}(Y) \int_{\mathbb{V}}\left(\frac{1}{(1+\gamma((\xi(t)), v))^{2}}-1\right) \tilde{N}(\mathrm{~d} t, \mathrm{~d} v) .
\end{aligned}
$$

Applying the generalized Itô's formula yields

$$
\begin{aligned}
\mathrm{d} V_{2}(Y) \leq & \left(1+V_{1}(Y)\right)^{\varrho-2}\left[\varrho \vartheta _ { k } ( 1 + V _ { 1 } ( Y ) ) \left(-2 V_{1}(Y)\left[r(k)-\frac{3}{2} \sigma^{2}(k)-\alpha(k) C_{0}(t)\right.\right.\right. \\
& \left.-\frac{1}{2} \int_{\mathbb{V}}\left(\frac{1}{(1+\gamma((k), v))^{2}}-1+2 \gamma((k), v)\right) \lambda(\mathrm{d} v)\right] \\
& \left.\left.+2 a_{1}(k) \prod_{0<t_{n}<t}\left(1+\delta_{n}\right) V_{1}^{1 / 2}(Y)\right)\right] \mathrm{d} t+2 \varrho(\varrho-1) \vartheta_{k} \sigma^{2}(k)\left(1+V_{1}(Y)\right)^{\varrho-2} V_{1}^{2}(Y) \\
& +\vartheta_{k} \int_{\mathbb{Y}}\left[\left(1+V_{1}(Y)+V_{1}(Y)\left(\frac{1}{(1+\gamma((k), v))^{2}}-1\right)\right)^{\varrho}-\left(1+V_{1}(Y)\right)^{\varrho}\right. \\
& \left.\left.-\varrho\left(1+V_{1}(Y)\right)^{\varrho-1} V_{1}(Y)\right)\left(\frac{1}{(1+\gamma((k), v))^{2}}-1\right)\right] \lambda(\mathrm{d} v) \mathrm{d} t \\
& +\sum_{l=1}^{N} \gamma_{k l} \vartheta_{l}\left(1+V_{1}(Y)\right)^{\varrho} \mathrm{d} t-2 \varrho \vartheta_{k} \sigma(k)\left(1+V_{1}(Y)\right)^{\varrho-1} V_{1}(Y) \mathrm{d} B(t) \\
& +\int_{\mathbb{V}}\left[\left(1+V_{1}(Y)+V_{1}(Y)\left(\frac{1}{(1+\gamma((k), v))^{2}}-1\right)\right)^{\varrho}-\left(1+V_{1}(Y)\right)^{\varrho}\right] \widetilde{N}(\mathrm{~d} t, \mathrm{~d} v) \\
\leq & \left(1+V_{1}(Y)\right)^{\varrho-2}\left\{-\left[\vartheta _ { k } \left(2 r(k) \varrho-\sigma^{2}(k) \varrho-2 \varrho \int_{\mathbb{Y}}(\gamma((k), v)) \lambda(\mathrm{d} v)-2 \alpha(k) C_{0}^{*}\right.\right.\right. \\
& \left.\left.-2 \varrho^{2} \sigma^{2}(k)\right)-\sum_{l=1}^{N} \gamma_{k l} \vartheta_{l}\right] V_{1}^{2}(Y)+2 a_{1}(k) M V_{1}^{3 / 2}(Y) \\
& -\left[\vartheta_{k}\left(2 r(k) \varrho-3 \sigma^{2}(k) \varrho-2 \varrho \int_{\mathbb{V}}(\gamma((k), v)) \lambda(\mathrm{d} v)-2 \alpha(k) C_{0}^{*}\right)-\sum_{l=1}^{N} \gamma_{k l} \vartheta_{l}\right] V_{1}(Y)
\end{aligned}
$$




$$
\begin{aligned}
& \left.+2 a_{1}(k) M V_{1}^{1 / 2}(Y)+\sum_{l=1}^{N} \gamma_{k l} \vartheta_{l}\right\} \mathrm{d} t-2 \varrho \vartheta_{k} \sigma(k)\left(1+V_{1}(Y)\right)^{\varrho-1} V_{1}(Y) \mathrm{d} B(t) \\
& +\int_{\mathbb{V}} \vartheta_{k}\left[\left(1+V_{1}(Y)+V_{1}(Y)\left(\frac{1}{(1+\gamma((k), v))^{2}}-1\right)\right)^{\varrho}-\left(1+V_{1}(Y)\right)^{\varrho}\right] \tilde{N}(\mathrm{~d} t, \mathrm{~d} v) \\
& =\left(1+V_{1}(Y)\right)^{\varrho-2}\left\{-\left(\vartheta_{k}\left(\varrho \bar{b}(k)-2 \varrho^{2} \sigma^{2}(k)\right)-\sum_{l=1}^{N} \gamma_{k l} \vartheta_{l}\right) V_{1}^{2}(Y)\right. \\
& +2 a_{1}(k) M V_{1}^{3 / 2}(Y)-\left(\vartheta_{k}\left(\varrho \bar{b}(k)-2 \varrho \sigma^{2}(k)\right)-\sum_{l=1}^{N} \gamma_{k l} \vartheta_{l}\right) V_{1}(Y) \\
& \left.+2 a_{1}(k) M V_{1}^{1 / 2}(Y)+\sum_{l=1}^{N} \gamma_{k l} \vartheta_{l}\right\} \mathrm{d} t-2 \varrho \vartheta_{k} \sigma(k)\left(1+V_{1}(Y)\right)^{\varrho-1} V_{1}(Y) \mathrm{d} B(t) \\
& +\int_{\mathbb{V}} \vartheta_{k}\left[\left(1+V_{1}(Y)+V_{1}(Y)\left(\frac{1}{(1+\gamma((k), v))^{2}}-1\right)\right)^{\varrho}-\left(1+V_{1}(Y)\right)^{\varrho}\right] \tilde{N}(\mathrm{~d} t, \mathrm{~d} v),
\end{aligned}
$$

and we use the following equation:

$$
\begin{aligned}
& \int_{\mathbb{V}} {\left[\left(1+V_{1}(Y)+V_{1}(Y)\left(\frac{1}{(1+\gamma((k), v))^{2}}-1\right)\right)^{\varrho}\right.} \\
&\left.-\left(1+V_{1}(Y)\right)^{\varrho}\right] \lambda(\mathrm{d} v)<0 .
\end{aligned}
$$

$$
\begin{aligned}
\mathrm{d} V_{3}(Y)= & \kappa e^{\kappa t} V_{2}(Y) \mathrm{d} t+e^{\kappa t} \mathrm{~d} V_{2}(Y) \leq e^{\kappa t}\left(1+V_{1}(Y)\right)^{\varrho-2}\left\{-\left(\vartheta_{k}\left(\varrho \bar{b}(k)-2 \varrho^{2} \sigma^{2}(k)\right)-\sum_{l=1}^{N} \gamma_{k l} \vartheta_{l}-\kappa \vartheta_{k}\right) V_{1}^{2}(Y)\right. \\
& +2 a_{1}(k) M V_{1}^{3 / 2}(Y)-\left(\vartheta_{k}\left(\varrho \bar{b}(k)-2 \varrho \sigma^{2}(k)\right)-\sum_{l=1}^{N} \gamma_{k l} \vartheta_{l}-2 \kappa \vartheta_{k}\right) V_{1}(Y) \\
& \left.+2 a_{1}(k) M V_{1}^{1 / 2}(Y)+\sum_{l=1}^{N} \gamma_{k l} \vartheta_{l}+\kappa \vartheta_{k}\right\} \mathrm{d} t-2 \varrho \vartheta_{k} e^{k t} \sigma(k)\left(1+V_{1}(Y)\right)^{\varrho-1} V_{1}(Y) \mathrm{d} B(t) \\
& +\vartheta_{k} e^{\kappa t} \int_{\mathbb{V}}\left[\left(1+V_{1}(Y)+V_{1}(Y)\left(\frac{1}{(1+\gamma(k), v)^{2}}-1\right)\right)^{\varrho}-\left(1+V_{1}(Y)\right)^{\varrho}\right] \tilde{N}(\mathrm{~d} t, \mathrm{~d} v) \\
= & e^{\kappa t} H(Y) \mathrm{d} t-2 \varrho \vartheta_{k} e^{\kappa t} \sigma(k)\left(1+V_{1}(Y)\right)^{\varrho-1} V_{1}(Y) \mathrm{d} B(t) \\
& +\vartheta_{k} e^{\kappa t} \int_{\mathbb{V}}\left[\left(1+V_{1}(Y)+V_{1}(Y)\left(\frac{1}{(1+\gamma(k), v)^{2}}-1\right)\right)^{\varrho}-\left(1+V_{1}(Y)\right)^{\varrho}\right] \tilde{N}(\mathrm{~d} t, \mathrm{~d} v),
\end{aligned}
$$

where

$$
\begin{aligned}
H(Y)= & \left(1+V_{1}(Y)\right)^{\varrho-2}\left\{-\left(\vartheta_{k}\left(\varrho \bar{b}(k)-2 \varrho^{2} \sigma^{2}(k)\right)-\sum_{l=1}^{N} \gamma_{k l} \vartheta_{l}-\kappa \vartheta_{k}\right) V_{1}^{2}(Y)+2 a_{1}(k) M V_{1}^{3 / 2}(Y)\right. \\
& \left.-\left(\vartheta_{k}\left(\varrho \bar{b}(k)-2 \varrho \sigma^{2}(k)\right)-\sum_{l=1}^{N} \gamma_{k l} \vartheta_{l}-2 \kappa \vartheta_{k}\right) V_{1}(Y)+2 a_{1}(k) M V_{1}^{1 / 2}(Y)+\sum_{l=1}^{N} \gamma_{k l} \vartheta_{l}+\kappa \vartheta_{k}\right\} .
\end{aligned}
$$


Since $H=\sup _{Y \in \mathbb{R}^{+}} H(Y)<+\infty$,

$$
\begin{aligned}
& \mathrm{d} V_{3}(Y) \leq e^{\kappa t} H \mathrm{~d} t-2 \varrho \vartheta_{k} e^{\kappa t} \sigma(k)\left(1+V_{1}(Y)\right)^{\varrho-1} V_{1}(Y) \mathrm{d} B(t) \\
& +\vartheta_{k} e^{k t} \int_{\mathbb{V}}\left[\left(1+V_{1}(Y)+V_{1}(Y)\left(\frac{1}{(1+\gamma(k), v)^{2}}-1\right)\right)^{\varrho}-\left(1+V_{1}(Y)\right)^{\varrho}\right] \tilde{N}(\mathrm{~d} t, \mathrm{~d} v) .
\end{aligned}
$$

Calculating (66), it is not difficult to show that

$$
\mathbb{E}\left[\vartheta_{k}\left(1+V_{1}(Y)\right)^{\varrho}\right] \leq \vartheta_{k} V_{2}\left(Y_{0}\right) \mathbb{E}\left[e^{-\kappa t}\right]+\frac{H}{\kappa} \mathbb{E}\left[1-e^{-\kappa t}\right] .
$$

Thus,

$$
\begin{aligned}
\limsup _{t \rightarrow+\infty} \mathbb{E}\left[Y(t)^{-2 \varrho}\right] & \leq \limsup _{t \longrightarrow+\infty} \mathbb{E}\left[\left(1+V_{1}(Y(t))\right)^{\varrho}\right] \\
& \leq \limsup _{t \longrightarrow+\infty}\left[\frac{V_{2}\left(Y_{0}\right)}{e^{\kappa t}}+\frac{H\left(1-e^{-\kappa t}\right)}{\kappa \min _{k \in \mathbb{M}}\left\{\vartheta_{k}\right\}}\right]=\bar{H} .
\end{aligned}
$$

Consequently,

$$
\begin{aligned}
& \limsup _{t \rightarrow+\infty} \mathbb{E}\left[X(t)^{-2 \varrho}\right] \\
& \quad=\limsup _{t \rightarrow+\infty} \mathbb{E}\left[\left(\prod_{0<t_{n}<t}\left(1+\delta_{n}\right)\right)^{-2 \varrho} Y(t)^{-2 \varrho}\right] \leq M^{-2 \varrho} \bar{H} .
\end{aligned}
$$

Then, for $\forall \varepsilon>0$, let $b_{1}=M(\varepsilon / \bar{H})^{1 / 2 \varrho}$; using Chebyshev's inequality, we have

$$
\begin{aligned}
\limsup _{t \rightarrow+\infty} \mathbb{P}\left\{|X(t)|<b_{1}\right\} & =\limsup _{t \rightarrow+\infty} \mathbb{P}\left\{|X(t)|^{-2 \varrho}>b_{1}^{-2 \varrho}\right\} \\
& \leq \lim _{t \rightarrow+\infty} \frac{\mathbb{E}\left[|X(t)|^{-2 \varrho}\right]}{b_{1}^{-2 \varrho}} \leq \varepsilon .
\end{aligned}
$$

In other words,

$$
\liminf _{t \longrightarrow+\infty} \mathbb{P}\left\{|X(t)| \geq b_{1}\right\} \geq 1-\varepsilon .
$$

Next, we prove that for $\forall \varepsilon>0$, there exists $b_{2}>0$ such that

$$
\liminf _{t \longrightarrow+\infty} \mathbb{P}\left\{X(t) \leq b_{2}\right\} \geq 1-\varepsilon .
$$

Applying generalized Itô's formula to (26):

$$
\begin{aligned}
\mathrm{d}(Y(t))= & Y(t)\left[r(\xi(t))-\alpha(\xi(t)) C_{0}(t)-a_{1}(\xi(t)) \prod_{0<t_{n}<t}\left(1+\delta_{n}\right) Y(t)\right. \\
& +a_{2}(\xi(t)) \prod_{0<t_{n}<t-\tau}\left(1+\delta_{n}\right) Y(t-\tau) \\
& \left.+a_{3}(\xi(t)) \int_{-\infty}^{0} \prod_{0<t_{n}<t+\theta}\left(1+\delta_{n}\right) Y(t+\theta) \mathrm{d} \varsigma(\theta)\right] \mathrm{d} t \\
& +\sigma(\xi(t)) Y(t) \mathrm{d} B(t)+Y\left(t^{-}\right) \int_{\mathbb{V}} \gamma((\xi(t)), v) \widetilde{N}(\mathrm{~d} t, \mathrm{~d} v) \\
\leq & {\left[\left(r(\xi(t))-\widehat{\alpha} C_{0}(t)\right) Y(t)-m \widehat{a}_{1} Y^{2}(t)+M \check{a}_{2} Y(t) Y(t-\tau)\right.} \\
& \left.+M \check{a}_{3} Y(t) \int_{-\infty}^{0} Y(t+\theta) \mathrm{d} \varsigma(\theta)\right] \mathrm{d} t+\sigma(\xi(t)) Y(t) \mathrm{d} B(t) \\
& +e^{t} Y\left(t^{-}\right) \int_{\mathbb{Y}} \gamma((\xi(t)), v) \widetilde{N}(\mathrm{~d} t, \mathrm{~d} v) \\
\leq & {\left[\left(r(\xi(t))-\widehat{\alpha} C_{0}(t)\right) Y(t)-m \widehat{a}_{1} Y^{2}(t)+\frac{M \check{a}_{2}}{2} Y^{2}(t)\right.} \\
& \left.+\frac{M \check{a}_{2}}{2} Y^{2}(t-\tau)+\frac{M \check{a}_{3}}{2} Y^{2}(t)+\frac{M \check{a}_{3}}{2} \int_{-\infty}^{0} Y^{2}(t+\theta) \mathrm{d} \varsigma(\theta)\right] \mathrm{d} t \\
& +\sigma(\xi(t)) Y(t) \mathrm{d} B(t)+Y\left(t^{-}\right) \int_{\mathbb{Y}} \gamma((\xi(t)), v) \tilde{N}(\mathrm{~d} t, \mathrm{~d} v) .
\end{aligned}
$$


14

Complexity

Thus,

$$
\begin{aligned}
\mathrm{d}\left(e^{t} Y(t)\right) \leq & e^{t}\left[\left(1+r(\xi(t))-\widehat{\alpha} C_{0}(t)\right) Y(t)-m \widehat{a}_{1} Y^{2}(t)+\frac{M \check{a}_{2}}{2} Y^{2}(t)\right. \\
& \left.+\frac{M \check{a}_{2}}{2} Y^{2}(t-\tau)+\frac{M \check{a}_{3}}{2} Y^{2}(t)+\frac{M \check{a}_{3}}{2} \int_{-\infty}^{0} Y^{2}(t+\theta) \mathrm{d} \varsigma(\theta)\right] \mathrm{d} t \\
& +e^{t} \sigma(\xi(t)) Y(t) \mathrm{d} B(t)+e^{t} Y\left(t^{-}\right) \int_{\mathbb{V}} \gamma((\xi(t)), v) \tilde{N}(\mathrm{~d} t, \mathrm{~d} v) \\
= & e^{t}\left[G(Y(t))-\frac{m \widehat{a}_{1}}{2} Y^{2}(t)+\frac{M \check{a}_{2}}{2} Y^{2}(t-\tau)+\frac{M \check{a}_{3}}{2} \int_{-\infty}^{0} Y^{2}(t+\theta) \mathrm{d} \varsigma(\theta)\right] \mathrm{d} t \\
& +e^{t} \sigma(\xi(t)) Y(t) \mathrm{d} B(t)+e^{t} Y\left(t^{-}\right) \int_{\mathbb{V}} \gamma((\xi(t)), v) \tilde{N}(\mathrm{~d} t, \mathrm{~d} v),
\end{aligned}
$$

where

$$
\begin{aligned}
G(Y(t))= & \left(1+r(\xi(t))-\widehat{\alpha} C_{0}(t)\right) Y(t) \\
& -\frac{m \widehat{a}_{1}}{2} Y^{2}(t)+\frac{M \check{a}_{2}}{2} Y^{2}(t)+\frac{M \check{a}_{3}}{2} Y^{2}(t) .
\end{aligned}
$$

According to $m \widehat{a}_{1}>M\left(\check{a}_{2}+\check{a}_{3}\right)$, we get

$$
G=\sup _{Y \in \mathbb{R}_{+}} G(Y)<+\infty \text {. }
$$

Then,

$$
\begin{aligned}
e^{t} Y(t)-Y(0) \leq & G\left(e^{t}-1\right)-\frac{m \widehat{a}_{1}}{2} \int_{0}^{t} e^{s} Y^{2}(s) \mathrm{d} s+\frac{M \check{a}_{2}}{2} \int_{0}^{t} e^{s} Y^{2}(s-\tau) \mathrm{d} s \\
& +\frac{M \check{a}_{3}}{2} \int_{0}^{t} e^{s} \int_{-\infty}^{0} Y^{2}(s+\theta) \mathrm{d} s(\theta) \mathrm{d} s+\int_{0}^{t} e^{s} \sigma(\xi(s)) Y(s) \mathrm{d} B(s) \\
& +\int_{0}^{t} e^{s} \int_{\mathbb{V}} \gamma((\xi(s)), v) Y\left(t^{-}\right) \tilde{N}(\mathrm{~d} s, \mathrm{~d} v) .
\end{aligned}
$$

On the one hand,

$$
\begin{aligned}
\frac{M \check{a}_{2}}{2} \int_{0}^{t} e^{s} Y^{2}(s-\tau) \mathrm{d} s= & \frac{M \check{a}_{2}}{2} \int_{-\tau}^{t-\tau} e^{s+\tau} Y^{2}(s) \mathrm{d} s \\
\leq & \frac{M \check{a}_{2}}{2} \int_{-\tau}^{0} e^{s+\tau} Y^{2}(s) \mathrm{d} s+\frac{M \check{a}_{2} e^{\tau}}{2} \int_{0}^{t} e^{s} Y^{2}(s) \mathrm{d} s, \\
& \cdot \frac{M \check{a}_{3}}{2} \int_{0}^{t} e^{s} \int_{-\infty}^{0} Y^{2}(s+\theta) \mathrm{d} \varsigma(\theta) \mathrm{d} s \\
= & \frac{M \check{a}_{3}}{2} \int_{0}^{t} e^{s}\left[\int_{-\infty}^{-s} Y^{2}(s+\theta) \mathrm{d} \varsigma(\theta)+\int_{-s}^{0} Y^{2}(s+\theta) \mathrm{d} \varsigma(\theta)\right] \mathrm{d} s \\
= & \frac{M \check{a}_{3}}{2} \int_{0}^{t} e^{s} \mathrm{~d} s \int_{-\infty}^{-s} e^{2 r(s+\theta)} Y^{2}(s+\theta) e^{-2 r(s+\theta)} \mathrm{d} \varsigma(\theta) \\
& +\frac{M \check{a}_{3}}{2} \int_{-t}^{0} \mathrm{~d} \varsigma(\theta) \int_{-\theta}^{t} e^{s} Y^{2}(s+\theta) \mathrm{d} s
\end{aligned}
$$




$$
\begin{aligned}
= & \frac{M \check{a}_{3}}{2} \int_{0}^{t} e^{s} \mathrm{~d} s \int_{-\infty}^{-s} e^{2 r(s+\theta)} Y^{2}(s+\theta) e^{-2 r(s+\theta)} \mathrm{d} \varsigma(\theta) \\
& +\frac{M \check{a}_{3}}{2} \int_{-t}^{0} \mathrm{~d} \varsigma(\theta) \int_{0}^{t+\theta} e^{(s-\theta)} Y^{2}(s) \mathrm{d} s \\
\leq & \frac{M \check{a}_{3}}{2}\|\xi\|_{C_{g}}^{2} \int_{0}^{t} e^{(1-2 r) s} \mathrm{~d} s \int_{-\infty}^{0} e^{-2 r \theta} \mathrm{d} \varsigma(\theta) \\
& +\frac{M \check{a}_{3}}{2} \int_{-\infty}^{0} e^{-\theta} \mathrm{d} \varsigma(\theta) \int_{0}^{t} e^{s} Y^{2}(s) \mathrm{d} s \\
\leq & \frac{M \check{a}_{3}}{2}\|\xi\|_{C_{g}}^{2} \rho_{r} t+\frac{M \check{a}_{3} \rho_{r}}{2} \int_{0}^{t} e^{s} Y^{2}(s) \mathrm{d} s .
\end{aligned}
$$

Therefore,

$$
\begin{aligned}
e^{t} Y(t) \leq & Y(0)+G\left(e^{t}-1\right)-\frac{m \widehat{a}_{1}-M\left(\check{a}_{2} e^{\tau}+\check{a}_{3} \rho_{r}\right)}{2} \int_{0}^{t} e^{s} Y^{2}(s) \mathrm{d} s \\
& +\frac{M \check{a}_{2}}{2} \int_{-\tau}^{0} e^{s+\tau} Y^{2}(s) \mathrm{d} s+\frac{M \check{a}_{3}}{2}\|\xi\|_{C_{g}}^{2} h_{r} t \\
& +\int_{0}^{t} e^{s} \sigma(\xi(s)) Y(s) \mathrm{d} B(s)+\int_{0}^{t} e^{s} \int_{\mathbb{V}} \gamma((\xi(s)), v) Y\left(t^{-}\right) \tilde{N}(\mathrm{~d} s, \mathrm{~d} v) .
\end{aligned}
$$

Taking expectations on both sides of (79) yields

$$
\underset{t \longrightarrow+\infty}{\limsup } \mathbb{E}[Y(t)] \leq G
$$

This leads to

$\limsup _{t \longrightarrow+\infty} \mathbb{E}[X(t)] \leq \limsup _{t \longrightarrow+\infty} \mathbb{E}\left[\prod_{0<t_{n}<t}\left(1+\delta_{n}\right) Y(t)\right] \leq M G$.

Then, for $\forall \varepsilon>0$, let $b_{2}=(M G / \varepsilon)$, and we have

$$
\limsup _{t \longrightarrow+\infty} \mathbb{P}\left\{|X(t)|>b_{2}\right\} \leq \lim _{t \longrightarrow+\infty} \frac{\mathbb{E}[|X(t)|]}{b_{2}}=\varepsilon \text {. }
$$

That is,

$$
\limsup _{t \rightarrow+\infty} \mathbb{P}\left\{|X(t)| \leq b_{2}\right\} \geq 1-\varepsilon
$$

From (71) and (83), $X(t)$ of system (25) is stochastically permanent.

\section{Asymptotic Properties}

Theorem 7. When A.1-A.3, A.5, and A.6 hold and any solution $X(t)$ of system (25) have the property that

$$
\underset{t \longrightarrow+\infty}{\limsup } \frac{\ln |X(t)|}{\ln t} \leq 1, \quad \text { a.s., }
$$

and if $\sum_{k=1}^{N} \pi_{k} \bar{b}(k)>0$,

$$
\liminf _{t \rightarrow+\infty} \frac{\ln |X(t)|}{\ln t} \geq-\frac{1}{2 \varrho}, \quad \text { a.s. }
$$

Proof. From (73), we have

$$
\begin{aligned}
\mathrm{d} Y(t)= & Y(t)\left[r(\xi(t))-\alpha(\xi(t)) C_{0}(t)-a_{1}(\xi(t)) \prod_{0<t_{n}<t}\left(1+\delta_{n}\right) Y(t)\right. \\
& +a_{2}(\xi(t)) \prod_{0<t_{n}<t-\tau}\left(1+\delta_{n}\right) Y(t-\tau) \\
& \left.+a_{3}(\xi(t)) \int_{-\infty}^{0} \prod_{0<t_{n}<t+\theta}\left(1+\delta_{n}\right) Y(t+\theta) \mathrm{d} \varsigma(\theta)\right] \mathrm{d} t \\
& +\sigma(\xi(t)) Y(t) \mathrm{d} B(t)+Y\left(t^{-}\right) \int_{\mathbb{Y}} \gamma((\xi(t)), v) \tilde{N}(\mathrm{~d} t, \mathrm{~d} v)
\end{aligned}
$$


Then,

$$
\begin{aligned}
\mathbb{E}\left[\limsup _{t \leq v \leq t+1} Y(v)\right] \leq & \mathbb{E}[Y(t)]+\mathbb{E}\left[\sup _{t \leq v \leq t+1}\left|\int_{t}^{t+1} Y(s) \mathrm{d} s\right|\right] \\
& +\mathbb{E}\left[\limsup _{t \leq v \leq t+1} \int_{t}^{v} \sigma(\xi(s)) Y(s) \mathrm{d} B(s)\right] \\
& +\mathbb{E}\left[\limsup _{t \leq v \leq t+1} \int_{t}^{v} \int_{\mathbb{V}} \gamma((\xi(s)), v) y(s) \tilde{N}(\mathrm{~d} s, \mathrm{~d} v)\right] .
\end{aligned}
$$

By the Burkholder-Davis-Gundy inequality (see [40]), one has

$$
\begin{gathered}
\mathbb{E}\left[\sup _{t \leq v \leq t+1} \int_{t}^{\nu} \sigma(\xi(s)) Y(s) \mathrm{d} B(s)\right] \\
\leq \sqrt{32} \mathbb{E}\left[\left(\int_{t}^{t+1} \sigma^{2}(\xi(s)) Y^{2}(s) \mathrm{d} s\right)^{1 / 2}\right] \\
\leq 4 \sqrt{2} \check{\sigma} \mathbb{E}\left[\sup _{t \leq \nu \leq t+1} Y(s)\right], \\
\mathbb{E}\left[\limsup _{t \leq v \leq t+1} \int_{t}^{v} \int_{\mathbb{Y}} \gamma((\xi(s)), v) Y(s) \tilde{N}(\mathrm{~d} s, \mathrm{~d} v)\right] \\
\leq \sqrt{32} \mathbb{E}\left[\int_{t}^{v} \int_{\mathbb{V}} \gamma^{2}((\xi(s)), v) Y^{2}(s) N(\mathrm{~d} s, \mathrm{~d} v)\right]^{1 / 2} \\
\leq 4 \sqrt{2}\left(\int_{\mathbb{\gamma}} \check{\gamma}^{2} \lambda(\mathrm{d} v)\right)^{1 / 2} \mathbb{E}\left[\sup _{t \leq \nu \leq t+1} Y(\nu)\right] .
\end{gathered}
$$

Substituting (80), (88), and (89) into (87) yields

$$
\mathbb{E}\left[\limsup _{t \leq \nu \leq t+1} Y(\nu)\right] \leq \mathbb{E}[Y(t)]+B \mathbb{E}\left[\sup _{t \leq \nu \leq t+1}[Y(\nu)]\right]
$$

where $B=G+4 \sqrt{2} \check{\sigma}+4 \sqrt{2}\left(\int_{\mathbb{\gamma}} \check{\gamma} \lambda(\mathrm{d} v)\right)^{1 / 2}$.

In view of (90), we have

$$
\mathbb{E}\left[\limsup _{t \leq \theta \leq t+1} Y(\nu)\right] \leq \bar{B}
$$

By Chebyshev's inequality, for $\forall \varepsilon>0$, we can obtain

$$
\mathbb{P}\left\{\omega \mid \sup _{\zeta \leq t \leq \zeta+1} Y(t)>\zeta^{1+\varepsilon}\right\} \leq \frac{\bar{B}}{\zeta^{1+\varepsilon}}, \quad \zeta=1,2, \ldots
$$

By using the Borel-Cantelli lemma [27], we get

$$
\sup _{\zeta \leq t \leq \zeta+1}[Y(t)] \leq \zeta^{1+\varepsilon}
$$

Therefore, for $\zeta \leq t \leq \zeta+1$ and $\zeta \geq \zeta_{0}(\omega)$, if $t \longrightarrow \infty$, then $\zeta \longrightarrow \infty$.

$$
\limsup _{t \longrightarrow+\infty} \frac{\ln |Y(t)|}{\ln t} \leq \frac{\ln \left(\sup _{\zeta \leq t \leq \zeta+1}[Y(t)]\right)}{\ln t} \leq \frac{\ln \zeta^{1+\varepsilon}}{\ln t} \leq 1+\varepsilon .
$$

Let $\varepsilon \longrightarrow 0$; then, $\limsup _{t \longrightarrow+\infty}(\ln Y(t) / \ln t) \leq 1$, that is, $\limsup _{t \longrightarrow+\infty} \frac{\ln |X(t)|}{\ln t}=\limsup _{t \longrightarrow+\infty} \frac{\sum_{0<t_{n}<t} \ln \left(1+\delta_{n}\right)+\ln |Y(t)|}{\ln t} \leq 1$.

Now, we prove the lower growth rate of the solution. From (68), we obtain

$$
\limsup _{t \longrightarrow+\infty} \mathbb{E}\left[\left(1+V_{1}(Y)\right)^{\varrho}\right] \leq \bar{H},
$$

where $\bar{H}$ is a positive constant.

Similarly,

$$
\begin{aligned}
\mathrm{d}\left[\left(1+V_{1}(Y)\right)^{\varrho}\right] \leq & \varrho\left(1+V_{1}(Y)\right)^{\varrho-2}\left[-2\left[r(\xi(t))-\frac{1}{2} \sigma^{2}(\xi(t))-\varrho \sigma^{2}(\xi(t))-\alpha(\xi(t)) C_{0}(t)\right.\right. \\
& \left.-\int_{\mathbb{V}}(\gamma((\xi(t)), v)) \lambda(\mathrm{d} v)\right] V_{1}^{2}(Y)+2 a_{1}(\xi(t)) \prod_{0<t_{n}<t}\left(1+\delta_{n}\right) V_{1}^{3 / 2}(Y) \\
& -2\left[r(\xi(t))-\frac{3}{2} \sigma^{2}(\xi(t))-\alpha(\xi(t)) C_{0}(t)-\int_{\mathbb{V}}(\gamma((\xi(t)), v)) \lambda(\mathrm{d} v)\right] V_{1}(Y) \\
& \left.+2 a_{1}(\xi(t)) \prod_{0<t_{n}<t}\left(1+\delta_{n}\right) V_{1}^{1 / 2}(Y)\right] \mathrm{d} t-2 \varrho \sigma(\xi(t))\left(1+V_{1}(Y)\right)^{\varrho-1} V_{1}(Y) \mathrm{d} B(t) \\
& +\int_{\mathbb{V}}\left[\left(1+V_{1}(Y)+V_{1}(Y)\left(\frac{1}{(1+\gamma((\xi(t)), v))^{2}}-1\right)\right)^{\varrho}-\left(1+V_{1}(Y)\right)^{\varrho}\right] \tilde{N}(\mathrm{~d} t, \mathrm{~d} v) \\
\leq & \varrho\left(1+V_{1}(Y)\right)^{\varrho-2}\left[D_{1} V_{1}^{2}(Y)+D_{2} V_{1}^{3 / 2}(Y)+D_{3} V_{1}(Y)+D_{2} V_{1}^{1 / 2}(Y)\right] \mathrm{d} t \\
& -2 \varrho \sigma(\xi(t))\left(1+V_{1}(Y)\right)^{\varrho-1} V_{1}(Y) \mathrm{d} B(t) \\
& +\int_{\mathbb{V}}\left[\left(1+V_{1}(Y)+V_{1}(Y)\left(\frac{1}{(1+\gamma((\xi(t)), v))^{2}}-1\right)\right)^{\varrho}-\left(1+V_{1}(Y)\right)^{\varrho}\right] \tilde{N}(\mathrm{~d} t, \mathrm{~d} v),
\end{aligned}
$$


where

$$
\begin{aligned}
& D_{1}=-2\left[r(\xi(t))-\frac{1}{2} \sigma^{2}(\xi(t))-\varrho \sigma^{2}(\xi(t))-\alpha(\xi(t)) C_{0}(t)-\int_{\mathbb{V}}(\gamma((\xi(t)), v)) \lambda(\mathrm{d} v)\right], \\
& D_{2}=2 M a_{1}(\xi(t)), \\
& D_{3}=-2\left[r(\xi(t))-\frac{3}{2} \sigma^{2}(\xi(t))-\alpha(\xi(t)) C_{0}(t)-\int_{\mathbb{V}}(\gamma((\xi(t)), v)) \lambda(\mathrm{d} v)\right] .
\end{aligned}
$$

Let $D>0$ be sufficiently large for

Then,

$$
\begin{aligned}
& \varrho\left(D_{1} V_{1}^{2}(Y)+D_{2} V_{1}^{3 / 2}(Y)+D_{3} V_{1}(Y)+D_{2} V_{1}^{1 / 2}(Y)\right) \\
& \quad \leq D\left(1+V_{1}(Y)\right)^{2} .
\end{aligned}
$$

$$
\begin{aligned}
\mathrm{d}\left[\left(1+V_{1}(Y)\right)^{\varrho}\right] \leq & D\left(1+V_{1}(Y)\right)^{\varrho}+2 \varrho \sigma(\xi(t))\left(1+V_{1}(Y)\right)^{\varrho-1} V_{1}(Y) \mathrm{d} B(t) \\
& +\int_{\mathbb{V}}\left[\left(1+V_{1}(Y)+V_{1}(Y)\left(\frac{1}{(1+\gamma((\xi(t)), v))^{2}}-1\right)\right)^{\varrho}-\left(1+V_{1}(Y)\right)^{\varrho}\right] \widetilde{N}(\mathrm{~d} t, \mathrm{~d} v) .
\end{aligned}
$$

Let $\zeta=1,2, \ldots$, and making use of (100) gives

$$
\begin{aligned}
& \mathbb{E}\left[\limsup _{(k-1) \zeta \leq t \leq k \zeta}\left(1+V_{1}(Y(t))\right)^{\varrho}\right] \\
& \leq \mathbb{E}\left[\left(1+V_{1}(Y((k-1) \zeta))\right)^{\varrho}\right]+D \mathbb{E}\left[\limsup _{(k-1) \zeta \leq t \leq k \zeta} \int_{(k-1) \zeta}^{t}\left(1+V_{1}(Y(t))\right)^{\varrho} \mathrm{d} s\right] \\
& +\mathbb{E}\left[\limsup _{(k-1) \zeta \leq t \leq k \zeta}\left|\int_{(k-1) \zeta}^{t} 2 \varrho \sigma(\xi(t))\left(1+V_{1}(Y)\right)^{\varrho-1} V_{1}(Y) \mathrm{d} B(t)\right|\right] \\
& +\mathbb{E}\left[\limsup _{(k-1) \zeta \leq t \leq k \zeta}\left|\int_{(k-1) \zeta}^{t} \varrho\left(1+V_{1}(Y)\right)^{\varrho-1} V_{1}(Y) \int_{\mathbb{Y}}\left(\frac{1}{(1+\gamma((\xi(t)), v))^{2}}-1\right) \tilde{N}(\mathrm{~d} t, \mathrm{~d} v)\right|\right]
\end{aligned}
$$

We compute that

$$
\begin{aligned}
D \mathbb{E}\left[\limsup _{(k-1) \zeta \leq t \leq k \zeta}\left|\int_{(k-1) \zeta}^{t}\left(1+V_{1}(Y(s))\right)^{\varrho} \mathrm{d} s\right|\right] & \leq D \mathbb{E}\left[\int_{(k-1) \zeta}^{t}\left|\left(1+V_{1}(Y(s))\right)^{\varrho}\right| \mathrm{d} s\right] \\
& \leq D \zeta \mathbb{E}\left[\limsup _{(k-1) \zeta \leq t \leq k \zeta}\left(1+V_{1}(Y(t))\right)^{\varrho}\right],
\end{aligned}
$$




$$
\begin{aligned}
\mathbb{E} & {\left[\limsup _{(k-1) \zeta \leq t \leq k \zeta}\left|\int_{(k-1) \zeta}^{t} 2 \varrho \sigma(\xi(t))\left(1+V_{1}(Y)\right)^{\varrho-1} V_{1}(Y) \mathrm{d} B(s)\right|\right] } \\
& \leq \sqrt{32} \mathbb{E}\left[\left(\int_{(k-1) \zeta}^{k \zeta} 4 \varrho^{2} \sigma^{2}(\xi(t))\left(1+V_{1}(Y)\right)^{2 \varrho-2} V_{1}^{2}(Y) \mathrm{d} s\right)^{1 / 2}\right] \\
& \leq 8 \sqrt{2} \varrho \check{\sigma} \mathbb{E}\left[\left(\int_{(k-1) \zeta}^{k \zeta}\left(1+V_{1}(Y)\right)^{2 \varrho} \mathrm{d} s\right)^{1 / 2}\right] \\
& \leq 8 \sqrt{2 \zeta} \varrho \check{\sigma} \mathbb{E}\left[\limsup _{(k-1) \zeta \leq t \leq k \zeta}\left(1+V_{1}(Y(t))\right)^{\varrho}\right], \\
\mathbb{E} & \left.\limsup _{(k-1) \zeta \leq t \leq k \zeta}\left|\int_{(k-1) \zeta}^{t} \varrho\left(1+V_{1}(Y)\right)^{\varrho-1} V_{1}(Y) \int_{\mathbb{V}}\left(\frac{1}{(1+\gamma((\xi(t)), v))^{2}}-1\right) \tilde{N}(\mathrm{~d} t, \mathrm{~d} v)\right|\right] \\
& \leq \sqrt{32} \mathbb{E}\left[\int_{(k-1) \zeta}^{t} \int_{\mathbb{V}} \varrho^{2}\left(1+V_{1}(Y)\right)^{2 \varrho-2} V_{1}^{2}(Y) \int_{\mathbb{V}}\left(\frac{1}{(1+\gamma((\xi(t)), v))^{2}}-1\right)^{2} N(\mathrm{~d} s, \mathrm{~d} v)\right]^{1 / 2} \\
& \leq \sqrt{32} \varrho\left(\int_{\mathbb{V}}\left(\frac{1}{(1+\widehat{\gamma})^{2}}-1\right)^{2} \lambda(\mathrm{d} v)\right)^{1 / 2} \mathbb{E}\left[\int_{(k-1) \zeta}^{t} \int_{\mathbb{V}}\left(1+V_{1}(Y)\right)^{2 \varrho} N(\mathrm{~d} s, \mathrm{~d} v)\right]^{1 / 2} \\
& \leq 4 \sqrt{2 \zeta} \varrho\left(\int_{\mathbb{V}}\left(\frac{1}{(1+\widehat{\gamma})^{2}}-1\right)^{2} \lambda(\mathrm{d} v)\right)^{1 / 2}\left[\sup _{(k-1) \zeta \leq t \leq k \zeta}\left(1+V_{1}(Y)\right)^{\varrho}\right] .
\end{aligned}
$$

Substituting (102)-(104) into (101) results in

$$
\begin{aligned}
& \mathbb{E}\left[\limsup _{(k-1) \zeta \leq t \leq k \zeta}\left(1+V_{1}(Y(t))\right)^{\varrho}\right] \\
& \leq \mathbb{E}\left[\left(1+V_{1}(Y((k-1) \zeta))\right)^{\varrho}\right] \\
& +C \mathbb{E}\left[\limsup _{(k-1) \zeta \leq t \leq k \zeta}\left(1+V_{1}(Y(t))\right)^{\varrho}\right],
\end{aligned}
$$

where

$C=D \zeta+8 \sqrt{2 \zeta} \varrho \check{\sigma}+4 \sqrt{2 \zeta} \varrho\left(\int_{\mathbb{Y}}\left(\left(1 /(1+\widehat{\gamma})^{2}\right)-1\right)^{2} \lambda(\mathrm{d} v)\right)^{1 / 2}$.

Then, from (96) and the above inequality, we get

$$
\mathbb{E}\left[\limsup _{(k-1) \zeta \leq t \leq k \zeta}\left(1+V_{1}(Y(t))\right)^{\varrho}\right] \leq \bar{C} .
$$

Then, for $\forall \varepsilon>0$, we have

$$
\begin{aligned}
& \mathbb{P}\left\{\omega \mid \sup _{(k-1) \zeta \leq t \leq k \zeta}\left(1+V_{1}(Y(t))\right)^{\varrho}>(k \zeta)^{1+\varepsilon}\right\} \\
& \quad \leq \frac{\bar{C}}{(k \zeta)^{1+\varepsilon}}, \quad k=1,2, \ldots
\end{aligned}
$$

Thus,

$$
\frac{\ln \left(1+V_{1}(Y(t))\right)^{\varrho}}{\ln t} \leq \frac{(1+\varepsilon) \ln (k \zeta)}{\ln ((k-1) \zeta)} .
$$

That is,

$$
\underset{t \longrightarrow+\infty}{\limsup } \frac{\ln \left(1+V_{1}(Y(t))\right)^{\varrho}}{\ln t} \leq 1+\varepsilon .
$$

Letting $\varepsilon \longrightarrow 0$ gives

$$
\limsup _{t \rightarrow+\infty} \frac{\ln \left(|Y(t)|^{-2 \varrho}\right)}{\ln t} \leq 1 \text {. }
$$

Consequently,

$$
\liminf _{t \longrightarrow+\infty} \frac{\ln (|Y(t)|)}{\ln t} \geq-\frac{1}{2 \varrho}
$$

That is,

$$
\liminf _{t \longrightarrow+\infty} \frac{\ln (|X(t)|)}{\ln t} \geq \liminf _{t \longrightarrow+\infty} \frac{\ln (m|Y(t)|)}{\ln t} \geq-\frac{1}{2 \varrho} .
$$

\section{Numerical Simulations}

Now, we use Matlab to analyze our results. We choose the same initial value $\left(X(0), C_{0}(0), C_{e}(0)\right)=(1,0.3,0.6)$ and the same parameter value as follows:

$$
\begin{aligned}
r(1) & =1, \\
\alpha(1) & =0.2, \\
\left(a_{1}(1), a_{2}(1), a_{3}(1)\right) & =(0.25,0.1,0.02), \\
r(2) & =0.8, \\
\alpha(2) & =0.3, \\
\left(a_{1}(2), a_{2}(2), a_{3}(2)\right) & =(0.3,0.03,0.05), \\
k & =0.6, \\
g & =0.26, \\
m & =0.05, \\
h & =0.23, \\
T & =4, \\
\tau_{1} & =2, \\
\lambda(\mathbb{Y}) & =1 .
\end{aligned}
$$




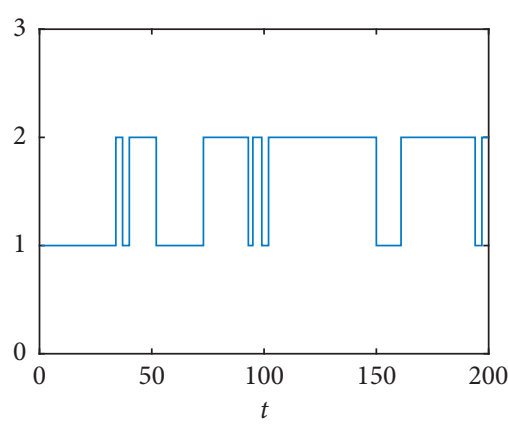

(a)

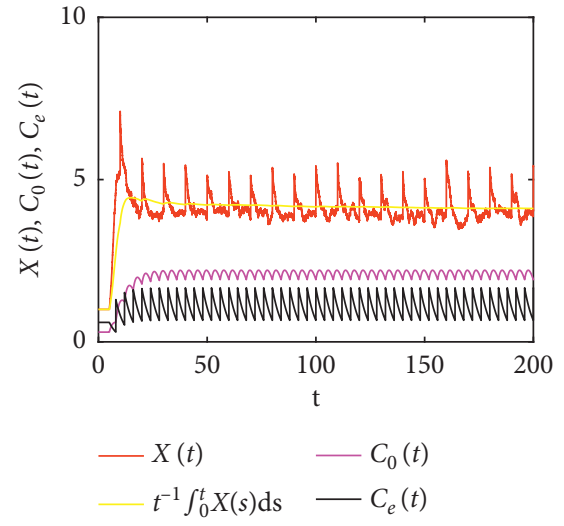

(b)

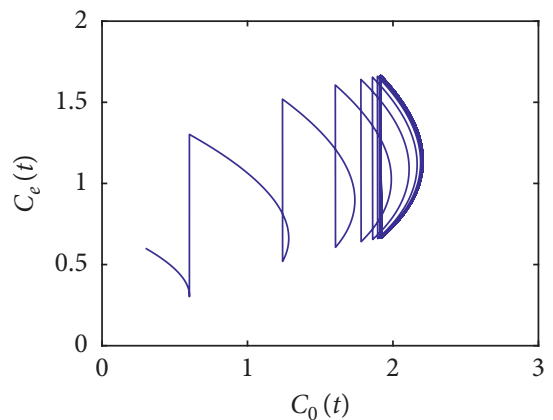

(c)

Figure 1: (a) Markov chains, (b) the time sequence diagram, and (c) the phase portrait of $C_{0}(t)$ and $C_{e}(t)$.
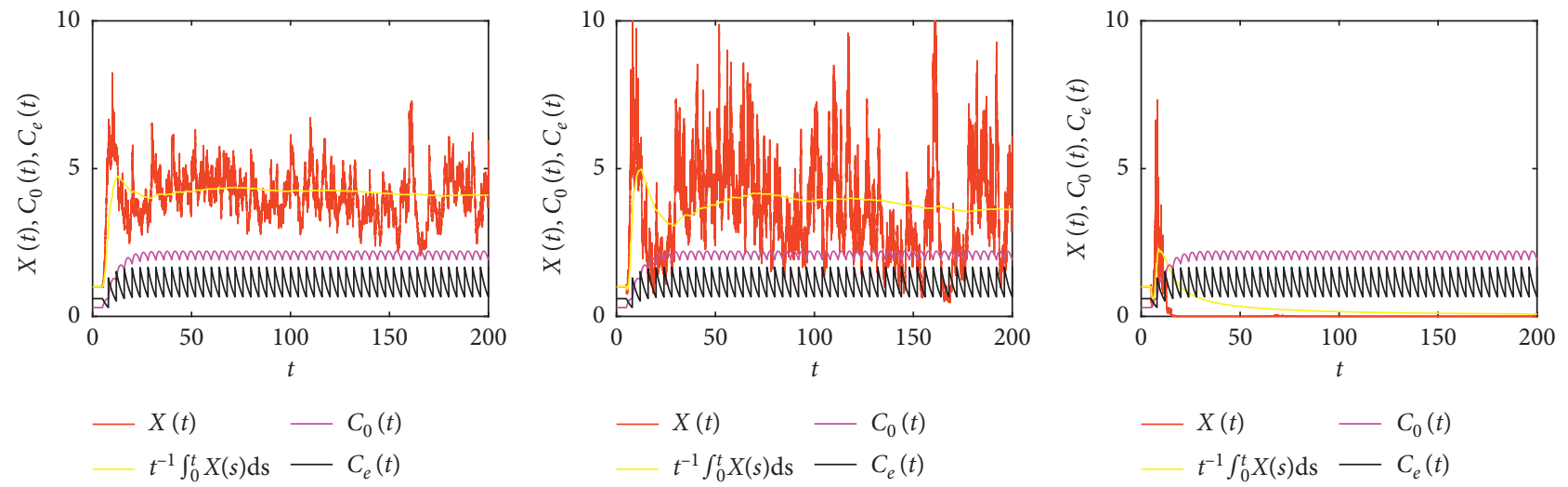

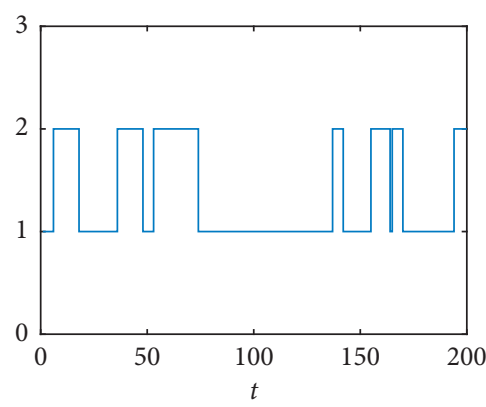

(a)

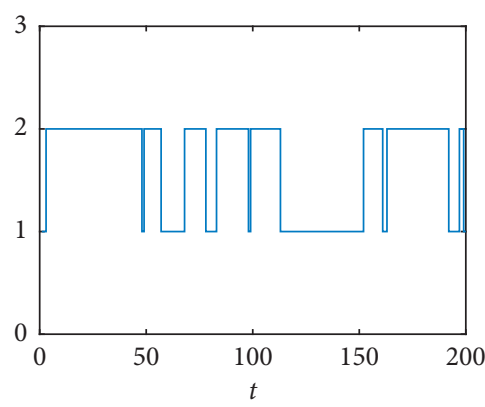

(b)

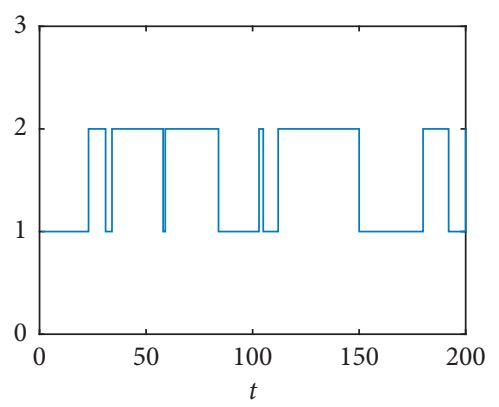

(c)

Figure 2: Effect of the white noise: (a) $\sigma(1)=0.2, \sigma(2)=0.2$; (b) $\sigma(1)=0.5, \sigma(2)=0.5$; (c) $\sigma(1)=1, \sigma(2)=1$.

In system (4), let $\xi(t) \in S=\{1,2\}$ and the generator

$$
\Gamma=\left(\begin{array}{cc}
-7 & 7 \\
3 & -3
\end{array}\right) \text {. }
$$

By solving (6), we get a stationary distribution $\pi=\left(\pi_{1}, \pi_{2}\right)=(0.3,0.7)$.

Now, we discuss the effects of different parameters on the population.

Example 1. Let $\gamma(1)=0.5, \gamma(2)=1.1, \sigma(1)=0.04, \sigma(2)=$ $0.1, u=1, \delta_{n}=e^{0.3}-1$. Then, we obtain $\eta^{*}=0.8771>$ $0.4208=\widehat{\alpha} \bar{C}_{0}$. According to Theorem 4, we know that $X(t)$ is weakly persistent (see Figure 1(a)). It can be seen from Figures 1(b) and 1(c) that the toxins in the organism $C_{0}(t)$ and the toxins in the environment $C_{e}(t)$ have a periodic solution.

6.1. Effect of the White Noise $\sigma$. Choosing the same parameters as in Example 1 but $\sigma$ : by comparing Figure 1(a) and Figures 2(a) and 2(b), we found that the disturbance of population in the stochastic model is positively correlated 

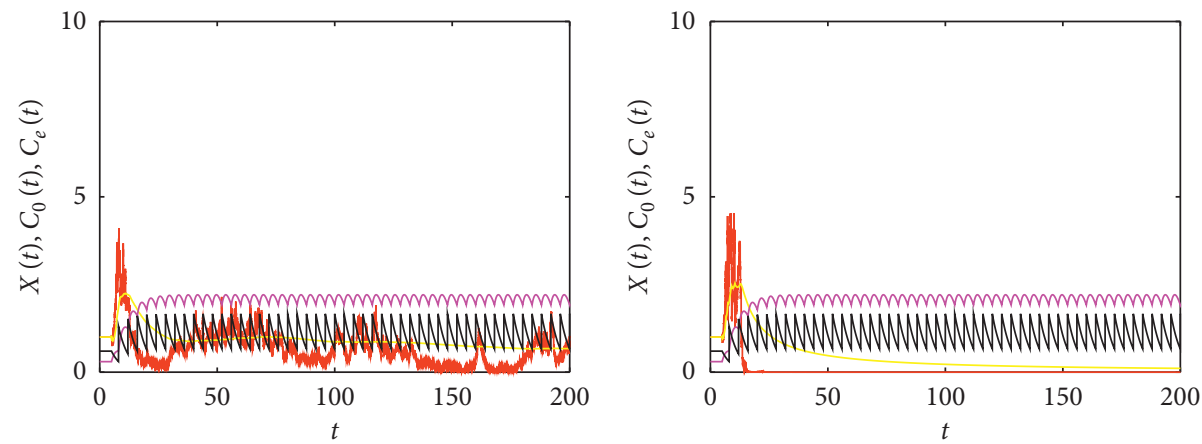

$\begin{array}{ll}X(t) & -C_{0}(t) \\ -t^{-1} \int_{0}^{t} X(s) \mathrm{d} s & -C_{e}(t)\end{array}$

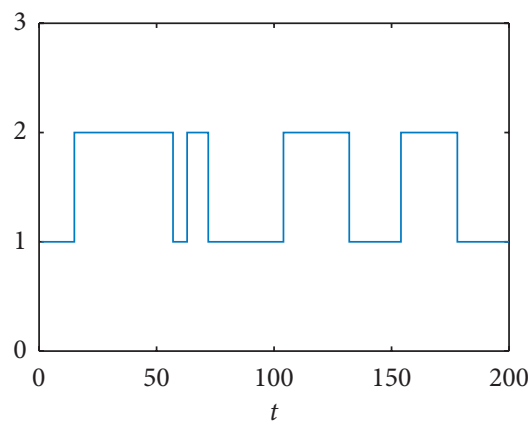

(a)
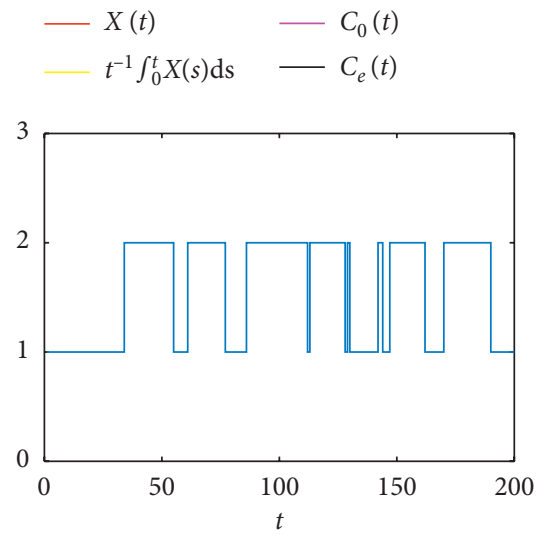

(b)

Figure 3: Effect of the Lévy noise: (a) $\gamma(1)=3, \gamma(2)=3$; (b) $\gamma(1)=10, \gamma(2)=10$.
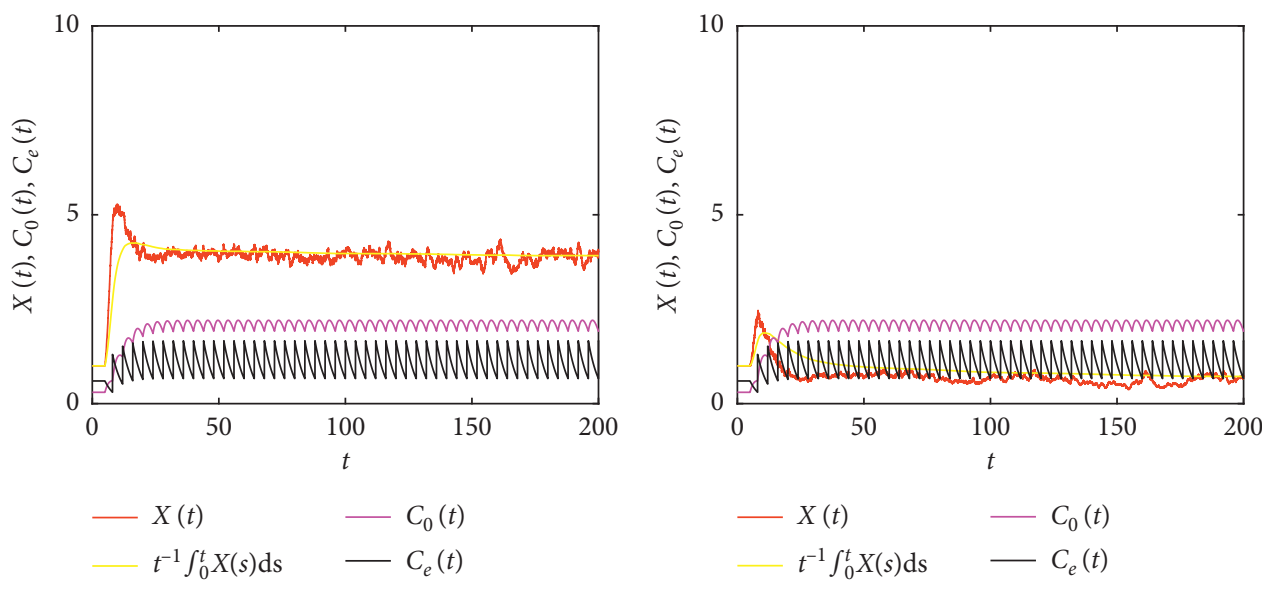

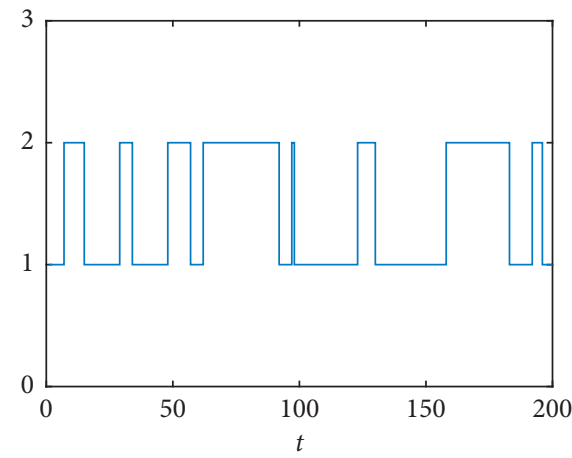

(a)

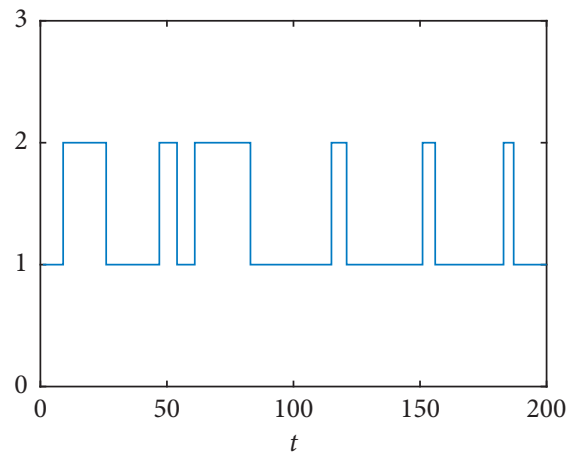

(b)

FIgURE 4: Effect of telegraph noise. 

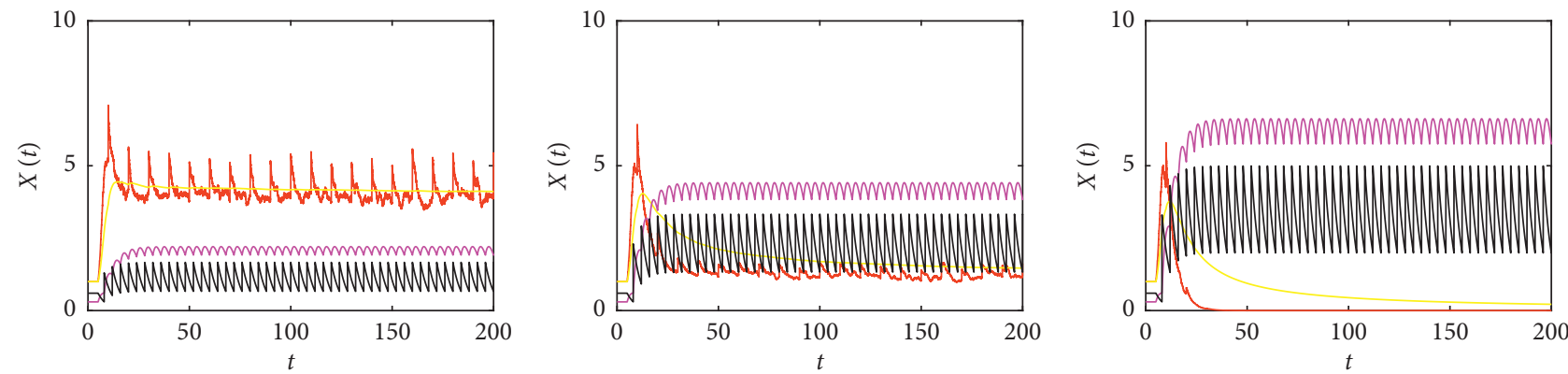
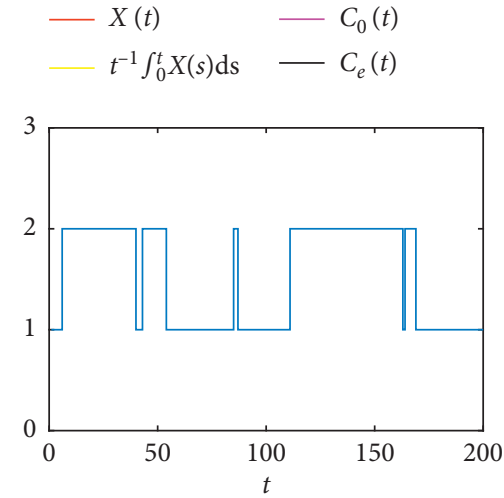

(a)
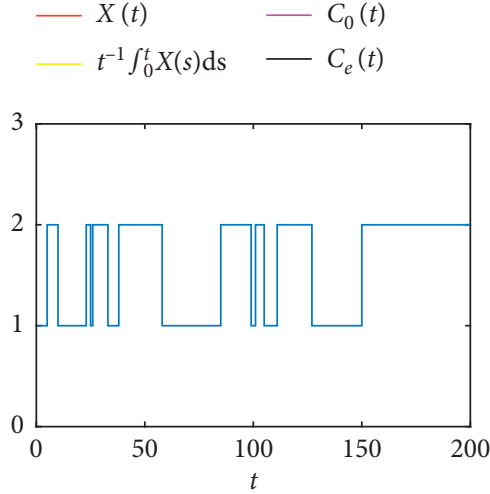

(b)
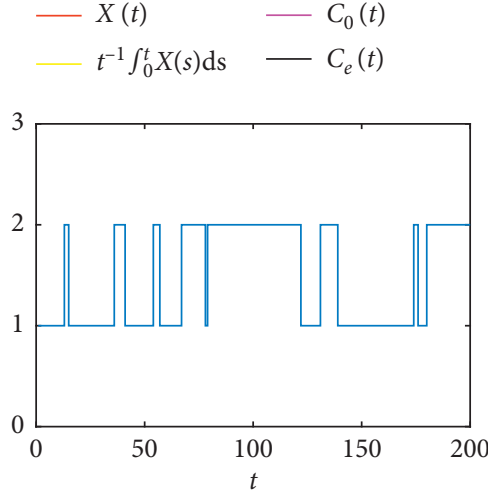

(c)

FiguRE 5: Effect of the exogenous total toxicant input: (a) $u=1$; (b) $u=2$; (c) $u=3$.
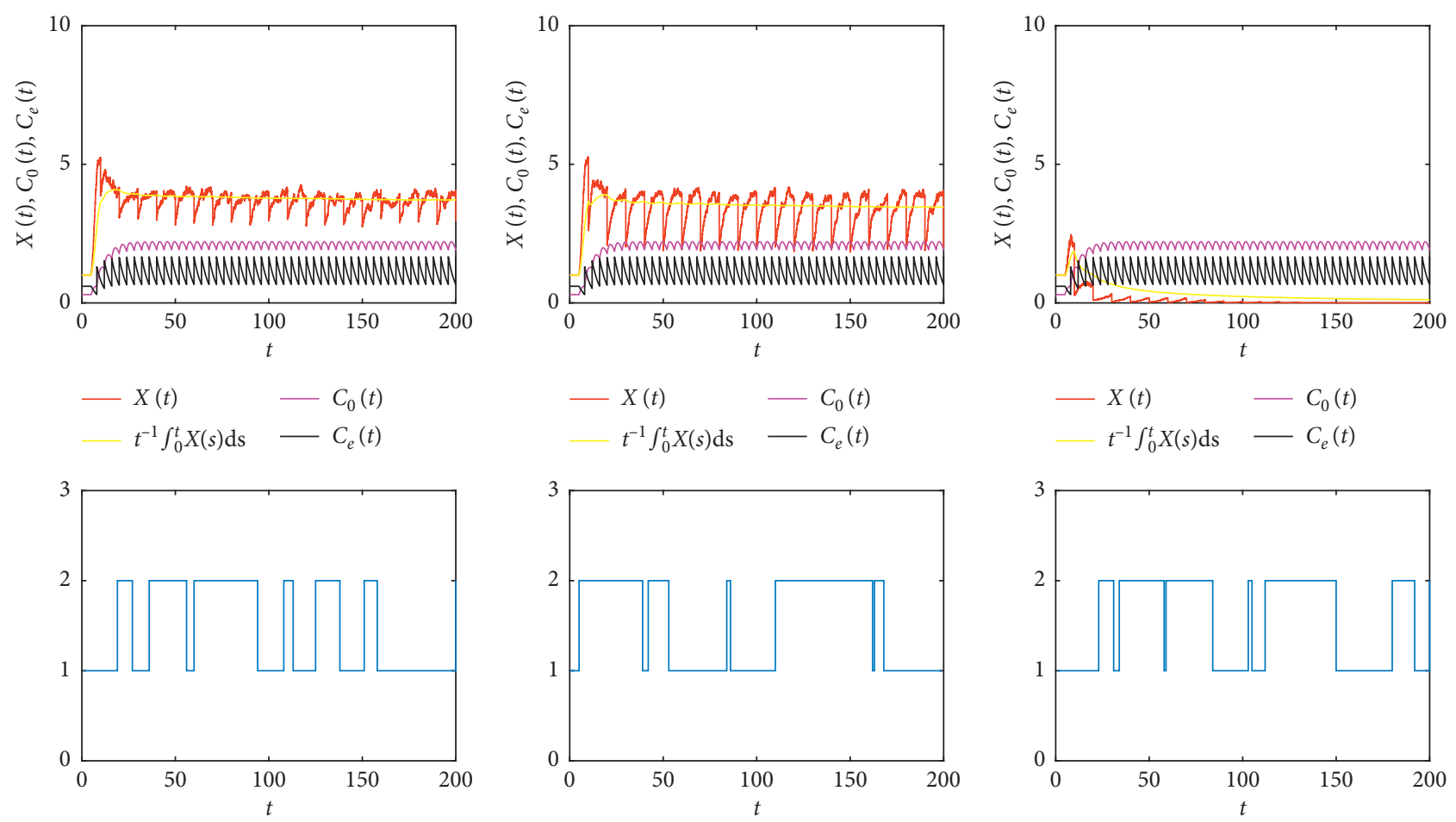

$\begin{array}{ll}X(t) & -C_{0}(t) \\ t^{-1} \int_{0}^{t} X(s) \mathrm{ds} & -C_{e}(t)\end{array}$

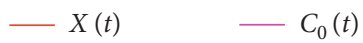

$t^{-1} \int_{0}^{t} X(s) \mathrm{d} s \quad-C_{e}(t)$

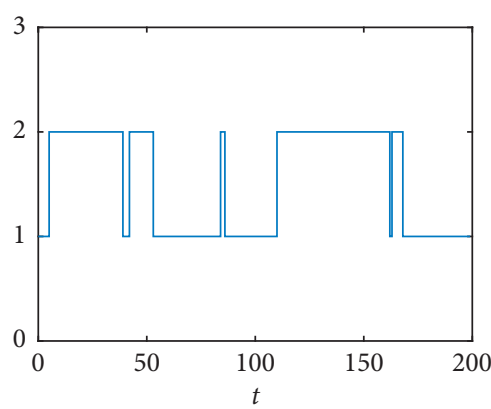

(b)

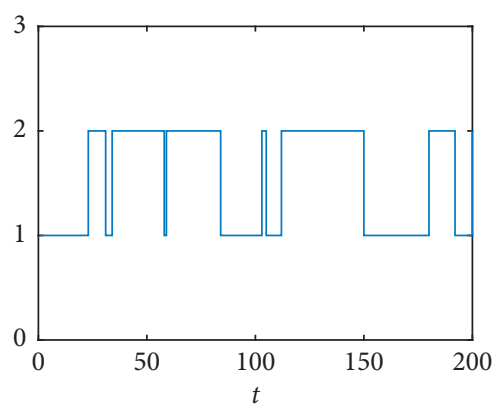

(c)

FIGURE 6: Effect of impulse: (a) $\delta_{n}=e^{-0.3}-1$; (b) $\delta_{n}=e^{-0.7}-1$; (c) $\delta_{n}=e^{-2}-1$. 
with the intensity of white noise. By comparing Figures 2(a)2 (c), the population will go extinct with increasing intensity of white noise. When the intensity of white noise is less than a certain level, the population still be persistent, while the white noise with large intensity may cause population extinction.

6.2. Effect of the Lévy Noise $\gamma$. Choosing the same parameters as in Example 1 but $\sigma(1)=0.4, \sigma(2)=0.4$, and $\gamma$ : from Figure 3 , it is concluded that the Lévy noise has a large impact on the persistence of population. It is shown that the number of the population will decrease when the intensity of the Lévy noise increases. When the intensity of the Lévy noise became larger, the population became extinct. Therefore, Lévy noise will not only reduce the number of species but also lead to population extinction.

6.3. Effect of Telegraph Noise. Choosing the same parameters as in Example 1 but $\delta_{n}=0$ : from Figure 4, we find that all parameter values are the same except the Markov chain. Since telegraph noise is described by a Markov chain, choosing a different Markov chain will produce different results.

6.4. Effect of the Exogenous Total Toxicant Input $u$. Choosing the same parameters as in Example 1 but $u$ : from Figure 5, we find that the population can still be persistent when the total input of exogenous toxicant is small (see Figures 5(a) and 5(b)), while the population will go extinct when the total input of exogenous toxicant is strong (see Figure 5(c)).

6.5. Effect of Impulse $\delta_{n}$. Choosing the same parameters as in Example 1 but $\delta_{n}$ : in Figure 6, we describe the impact of pulse harvest on the population. By comparing Figures 6(a)$6(c)$, we find that with the increase of the harvest, the population will gradually decrease until it becomes completely extinct.

\section{Conclusion}

In this paper, we explore the dynamics of a stochastic delay hybrid logistic model with two-pulse perturbations. First, by using Itô's formula, exponential martingale inequality, Chebyshev's inequality, and other mathematical skills, we establish some sufficient conditions for extinction, nonpersistence in the mean, weak persistence, persistence in the mean, and stochastic permanence. Then, the asymptotic properties of the lower growth rate and the upper growth rate of the solution are estimated.

Now, we give the key results as follows:

(I) (1) If $\eta^{*}<\widehat{\alpha} \bar{C}_{0}$, then the population $X(t)$ is extinct.

(2) If $\eta^{*}=\widehat{\alpha} \bar{C}_{0}$, then the population $X(t)$ is nonpersistent in the mean.

(3) If $\eta^{*}>\widehat{\alpha} \bar{C}_{0}$, then the population $X(t)$ is weakly persistent.
(4) If $\eta_{*}>\check{\alpha} \bar{C}_{0}$, then the population $X(t)$ is persistent in the mean.

(5) If $\sum_{k=1}^{N} \pi_{k} \bar{b}(k)>0$, then the population $X(t)$ is stochastically permanent.

(II) The solution $X(t)$ obeys

$$
\begin{aligned}
\underline{*} & =\frac{\eta_{*}-\check{\alpha} \bar{C}_{0}}{\check{a}_{1}} \leq \liminf _{t \longrightarrow+\infty} \frac{1}{t} \int_{0}^{t} X(s) \mathrm{d} s \\
& \leq \limsup _{t \longrightarrow+\infty} \frac{1}{t} \int_{0}^{t} X(s) \mathrm{d} s \leq \frac{\eta^{*}-\widehat{\alpha} \bar{C}_{0}}{a}=\bar{X}^{*}, \quad \text { a.s. }
\end{aligned}
$$

(III) The solution satisfies

$$
\begin{aligned}
& \limsup _{t \longrightarrow+\infty} \frac{\ln |X(t)|}{\ln t} \leq 1, \\
& \liminf _{t \rightarrow+\infty} \frac{\ln |X(t)|}{\ln t} \geq-\frac{1}{2 \varrho}, \quad \text { a.s. }
\end{aligned}
$$

From our results and analysis, we can obtain the following conclusions. (1) Both white noise and Lévy noise tend to have negative effects on the persistence of population. However, the Lévy jump may have a greater influence than white noise on the persistence of population. (2) If the choice of telegraph noise is different, the persistence of the population will produce different results. (3) The total input of exogenous toxicant has a great disadvantaged influence on persistence of the population. With the increase of the total input of exogenous toxicant, the number of population will decrease, which enlightens us to reduce pollutant emissions to protect the ecological system. (4) The pulse release of the population is beneficial to the growth of the population; the pulse harvest of the population is beneficial to the growth of the population under reasonable conditions, but once overharvested, it will cause the population to become extinct.

These give us significant enlightenment: (1) when fishermen are fishing, they must maintain reasonable fishing to avoid overfishing that can lead to population extinction; (2) the discharge of factory sewage, exhaust gas, domestic sewage, etc., should be strictly controlled, and we should do our best to reduce the discharge of polluted toxins; and (3) humans should reduce interference with populations.

\section{Data Availability}

No data were used to support this study.

\section{Conflicts of Interest}

The authors declare that they have no conflicts of interest.

\section{Acknowledgments}

This study was supported by the Research Fund for the Taishan Scholar Project of Shandong Province of China, the Shandong Provincial Natural Science Foundation of China 
(ZR2019MA003), the National Natural Science Foundation of China (11901360), and the SDUST Innovation Fund for Graduate Students (SDKDYC190119).

\section{References}

[1] Q. Liu, D. Jiang, T. Hayat, and A. Alsaedi, "Long-time behavior of a stochastic logistic equation with distributed delay and nonlinear perturbation," Physica A: Statistical Mechanics and Its Applications, vol. 508, pp. 289-304, 2018.

[2] D. Li, J. a. Cui, and G. Song, "Permanence and extinction for a single-species system with jump-diffusion," Journal of Mathematical Analysis and Applications, vol. 430, no. 1, pp. 438-464, 2015.

[3] B. Yang, Y. Cai, K. Wang, and W. Wang, "Optimal harvesting policy of logistic population model in a randomly fluctuating environment," Physica A: Statistical Mechanics and Its Applications, vol. 526, Article ID 120817, 2019.

[4] Y. Xu, S. Gao, and D. Chen, "Persistence and extinction of a nonautonomous switching single-species population model," Applied Mathematics Letters, vol. 103, Article ID 106187, 2019.

[5] Y. Lv, L. Chen, and F. Chen, "Stability and bifurcation in a single species logistic model with additive Allee effect and feedback control," Advances in Difference Equations, vol. 2020, no. 1, p. 129, 2020.

[6] C. Xu, "Phenomenological bifurcation in a stochastic logistic model with correlated colored noises," Applied Mathematics Letters, vol. 101, Article ID 106064, 2020.

[7] M. Liu and K. Wang, "Persistence and extinction in stochastic non-autonomous logistic systems," Journal of Mathematical Analysis and Applications, vol. 375, no. 2, pp. 443-457, 2011.

[8] E. Beretta and Y. Takeuchi, "Global stability of single-species diffusion Volterra models with continuous time delays," Bulletin of Mathematical Biology, vol. 49, no. 4, pp. 431-448, 1987.

[9] M. Liu and K. Wang, "On a stochastic logistic equation with impulsive perturbations," Computers \& Mathematics with Applications, vol. 63, no. 5, pp. 871-886, 2012.

[10] S. Zhao, S. Yuan, and H. Wang, "Threshold behavior in a stochastic algal growth model with stoichiometric constraints and seasonal variation," Journal of Differential Equations, vol. 268, no. 9, pp. 5113-5139, 2020.

[11] X. Yu and S. Yuan, "Asymptotic properties of a stochastic chemostat model with two distributed delays and nonlinear perturbation," Discrete \& Continuous Dynamical Systems-B, vol. 25, no. 7, pp. 2373-2390, 2020.

[12] W. Zhou, J. Yang, X. Yang, A. Dai, H. Liu, and J. a. Fang, "pth moment exponential stability of stochastic delayed hybrid systems with Lévy noise," Applied Mathematical Modelling, vol. 39, no. 18, pp. 5650-5658, 2015.

[13] X. Yu, S. Yuan, and T. Zhang, "Asymptotic properties of stochastic nutrient-plankton food chain models with nutrient recycling," Nonlinear Analysis: Hybrid Systems, vol. 34, pp. 209-225, 2019.

[14] S. Yuan, D. Wu, G. Lan, and H. Wang, "Noise-induced transitions in a nonsmooth producer-Grazer model with stoichiometric constraints," Bulletin of Mathematical Biology, vol. 82, no. 5, p. 55, 2020.

[15] X. Sun, W. Zuo, D. Jiang, and T. Hayat, "Unique stationary distribution and ergodicity of a stochastic logistic model with distributed delay," Physica A: Statistical Mechanics and Its Applications, vol. 512, pp. 864-881, 2018.

[16] S. Ma and D. Dong, "The asymptotic stability analysis in stochastic logistic model with Poisson growth coefficient,"
Theoretical and Applied Mechanics Letters, vol. 4, no. 1, Article ID 013004, 2014.

[17] N. Kaplan, "A continuous time Markov branching model with random environments," Advances in Applied Probability, vol. 5, no. 1, pp. 37-54, 1973.

[18] N. H. Du, R. Kon, K. Sato, and Y. Takeuchi, "Dynamical behavior of Lotka-Volterra competition systems: non-autonomous bistable case and the effect of telegraph noise," Journal of Computational and Applied Mathematics, vol. 170, no. 2, pp. 399-422, 2004.

[19] M. Slatkin, "The dynamics of a population in a Markovian environment," Ecology, vol. 59, no. 2, pp. 249-256, 1978.

[20] A. Settati and A. Lahrouz, "Stationary distribution of stochastic population systems under regime switching," Applied Mathematics and Computation, vol. 244, pp. 235-243, 2014.

[21] X. Li, A. Gray, D. Jiang, and X. Mao, "Sufficient and necessary conditions of stochastic permanence and extinction for stochastic logistic populations under regime switching," Journal of Mathematical Analysis and Applications, vol. 376, no. 1, pp. 11-28, 2011.

[22] T. Zhang and H. Chen, "The stability with a general decay of stochastic delay differential equations with Markovian switching," Applied Mathematics and Computation, vol. 359, no. 9, pp. 294-307, 2019.

[23] Y. Cai, S. Cai, and X. Mao, "Stochastic delay foraging arena predator-prey system with Markov switching," Stochastic Analysis and Applications, vol. 38, no. 2, pp. 191-212, 2020.

[24] X. Li, D. Jiang, and X. Mao, "Population dynamical behavior of Lotka-Volterra system under regime switching," Journal of Computational and Applied Mathematics, vol. 232, no. 2, pp. 427-448, 2009.

[25] X. Yu, S. Yuan, and T. Zhang, "Persistence and ergodicity of a stochastic single species model with Allee effect under regime switching," Communications in Nonlinear Science and Numerical Simulation, vol. 59, pp. 359-374, 2018.

[26] M. Gao, D. Jiang, and T. Hayat, "The threshold of a chemostat model with single-species growth on two nutrients under telegraph noise," Communications in Nonlinear Science and Numerical Simulation, vol. 75, pp. 160-173, 2019.

[27] X. Mao and C. Yuan, Stochastic Differential Equations with Markovian Switching, Imperial College Press, London, UK, 2006.

[28] X. Li and G. Yin, "Logistic models with regime switching: permanence and ergodicity," Journal of Mathematical Analysis and Applications, vol. 441, no. 2, pp. 593-611, 2016.

[29] M. Liu and K. Wang, "Dynamics and simulations of a logistic model with impulsive perturbations in a random environment," Mathematics and Computers in Simulation, vol. 92, pp. 53-75, 2013.

[30] Y. Deng and M. Liu, "Analysis of a stochastic tumor-immune model with regime switching and impulsive perturbations," Applied Mathematical Modelling, vol. 78, pp. 482-504, 2020.

[31] H. Lv, Z. Liu, Z. Li, L. Wang, and D. Xu, "Two impulsive stochastic delay single-species models incorporating Lévy noise," Journal of Applied Mathematics and Computing, vol. 58, no. 1-2, pp. 721-753, 2018.

[32] C. Lu and X. Ding, "Persistence and extinction of a stochastic logistic model with delays and impulsive perturbation," Acta Mathematica Scientia, vol. 34, no. 5, pp. 1551-1570, 2014.

[33] C. Lu, B. Li, L. Zhou, and L. Zhang, "Survival analysis of an impulsive stochastic delay logistic model with Lévy jumps," Mathematical Biosciences and Engineering, vol. 16, no. 5, pp. 3251-3271, 2019. 
[34] C. Lu, Q. Ma, and X. Ding, "Persistence and extinction for stochastic logistic model with Lévy noise and impulsive perturbation," Electronic Journal of Differential Equations, vol. 2015, no. 247, pp. 1-14, 2015.

[35] D. Li, T. Guo, and Y. Xu, "The effects of impulsive toxicant input on a single-species population in a small polluted environment," Mathematical Biosciences and Engineering, vol. 16, no. 6, pp. 8179-8194, 2019.

[36] M. Liu and K. Wang, "Persistence and extinction of a singlespecies population system in a polluted environment with random perturbations and impulsive toxicant input," Chaos, Solitons \& Fractals, vol. 45, no. 12, pp. 1541-1550, 2012.

[37] Y. Liu, Q. Liu, and Z. Liu, "Dynamical behaviors of a stochastic delay logistic system with impulsive toxicant input in a polluted environment," Journal of Theoretical Biology, vol. 329, pp. 1-5, 2013.

[38] B. Liu, L. Chen, and Y. Zhang, "The effects of impulsive toxicant input on a population in a polluted environment," Journal of Biological Systems, vol. 11, no. 3, pp. 265-274, 2003.

[39] J. K. Hale and J. Kato, "Phase space for retarded equations with infinite delay," Funkcialaj Ekvacioj-Serio Internacia, vol. 21, pp. 11-41, 1978.

[40] X. Mao, Stochastic Differential Equations and Applications, Horwood Publishing, Chichester, UK, 1997.

[41] R. Lipster, "A strong law of large numbers for local martingales," Stochastics, vol. 3, pp. 217-228, 1980. 